COMMUNICATIONS IN

ANALYSIS AND GEOMETRY

Volume 14, Number 3, 497-549, 2006

\title{
Harmonic maps from 2-complexes
}

\author{
Georgios Daskalopoulos ${ }^{1}$ \\ BROWN UNIVERSITY \\ daskal@math. brown.edu \\ CHIKAKo Mese ${ }^{2}$ \\ JOHNS HOPKINS UNIVERSITY \\ cmese@math.jhu.edu
}

\begin{abstract}
We develop the theory of harmonic maps from a flat admissible 2complex into a metric space of non-positive curvature. As an application, we give a harmonic maps analysis of the Morgan-Shalen compactification of $S L(2, \mathbf{C})$ representations of a finitely generated group.
\end{abstract}

\section{Introduction.}

Let $\operatorname{Isom}_{+}\left(\mathbf{H}^{3}\right)$ denote the Lie group of orientation-preserving isometries of $\mathbf{H}^{3}$ and $\Gamma$ be a discrete group. Any discrete and faithful representation $\rho$ : $\Gamma \rightarrow$ Isom $_{+}\left(\mathbf{H}^{3}\right)$ gives rise to a hyperbolic manifold $\mathbf{H}^{3} / \rho(\Gamma)$ and an isomorphism $\Gamma \approx \pi_{1}\left(\mathbf{H}^{3} / \rho(\Gamma)\right)$ that is well defined up to conjugation. The space $\mathcal{H}^{3}(\Gamma)$ of conjugacy classes of discrete and faithful representations $\rho: \Gamma \rightarrow$ Isom $_{+}\left(\mathbf{H}^{3}\right)$ can be compactified by projective limits of length functions associated to the representations where the length function $l_{\rho_{k}}: \Gamma \rightarrow \mathbf{R}^{+}$of $\rho_{k}: \Gamma \rightarrow \operatorname{Isom}_{+}\left(\mathbf{H}^{3}\right)$ is

$$
l_{\rho_{k}}(g)=\inf _{z \in \mathbf{H}^{3}} d_{\mathbf{H}^{3}}\left(z, \rho_{k}(g) z\right) .
$$

These limits turn out to be projectively equivalent to the length functions of isometric actions of $\Gamma$ on $\mathbf{R}$-trees. Called the Morgan-Shalen compactification, this is the "tree-theoretic" approach to Thurston's Bounded Image Theorem, a part of the Thurston hyperbolization program, developed in [CuSh] [MoSh1] [Mo] [MoSh2] [MoSh3]. See also the expositions in [Ka] and [Ot]. The analogous two dimensional theory is equivalent to Thurston's compactification of the Teichmüller space of a surface.

\footnotetext{
${ }^{1}$ supported by research grant NSF DMS-02041912

${ }^{2}$ supported by research grant NSF DMS-0072483
} 
In [DDW1] and [DDW2], harmonic maps is used to study the MorganShalen compactification using equivariant harmonic maps from Riemannian manifolds into $\mathbf{H}^{3}$. In particular, for any unbounded sequence of irreducible $S L(2, \mathbf{C})$ representations of the fundamental group $\Gamma$ of a compact Riemannian manifold $M$ without boundary, they produce an $\mathbf{R}$-tree $T$ along with an equivariant harmonic map $u: \tilde{M} \rightarrow T$. The method in [DDW1] and [DDW2] is based on the theory of harmonic maps from Riemannian manifolds into singular spaces of nonpositive curvature (NPC spaces) developed by Gromov-Schoen [GS], Korevaar-Schoen [KS1] [KS2] [KS3] and Jost [Jo]. However, from the point of view of combinatorial group theory and three dimensional topology, one would like to understand finitely generated group from their actions on $\mathbf{R}$-trees. In many cases, these actions are "geometric" which means that the corresponding trees are associated to leaf spaces of measured foliations (or laminations) on two dimensional complexes. This is a generalization of the familiar picture of measured foliations on surfaces studied in the Thurston theory. For more details from the topological viewpoint, we refer to $[\mathrm{BesF}],[\mathrm{Ka}],[\mathrm{LePa}][\mathrm{Ot}]$ for example. The motivation for this paper and its sequel is to bring harmonic map theory and holomorphic quadratic differentials into the study of finitely generated groups.

Let $\Gamma$ be a finitely generated group acting on $T$, i.e. there is a homomorphism $\rho: \Gamma \rightarrow \operatorname{Isom}(T)$. We then consider a finite 2-complex $X$ and a normal covering $p: \bar{X} \rightarrow X$ with transformation group $\Gamma$. If $\Gamma$ is finitely presented, then we may take $X$ so that $\pi_{1}(X)=\Gamma$ and $p: \tilde{X} \rightarrow X$ the universal cover, but in general, $\bar{X}$ may fail to be simply connected. We may assume that $X$ is an admissible finite 2-complex without boundary (see Section 2.2). Moreover, we assign Riemannian metrics on faces of $X$ so that $X$ is a flat admissible finite 2-complex without boundary as in Section 3. (See Sections 2.1 and 2.2 for precise definitions.) We study the existence, regularity and compactness properties of equivariant harmonic map $f$ from a flat admissible finite 2-complex into $\mathbf{R}$-trees. Here, equivariant means that the map honors the action of $\Gamma$ on Isom $(T)$, i.e $f(\gamma x)=\rho(\gamma)(f(x))$. We then apply this to study the MorganShalen compactification of the $S L(2, \mathbf{C})$ character variety of any finitely generated group. More precisely, our main results are as follows.

Theorem 1.1 (Regularity). Let $f: X \rightarrow Y$ be a harmonic map from a flat admissible 2-complex without boundary to a NPC space. Then $f$ is locally Lipschitz continuous away from the vertices of $X$ with Lipschitz constant dependent only on the total energy of $f$ and the distance away from the vertices. Furthermore, $f$ is globally Hölder continuous with Hölder constant and exponent only dependent on the total energy of $f$. 
Theorem 1.2 (Compactness). If $f_{k}: X \rightarrow\left(Y_{k}, d_{k}\right)$ is a sequence of equivariant harmonic maps into NPC spaces with bounded energies, then there exists a subsequence which converges to an equivariant harmonic map $f_{\infty}$ : $X \rightarrow\left(Y_{\infty}, d_{\infty}\right)$ (in the sense of $\left.[K S 1]\right)$.

The compactness theorem allows us to repeat the construction of [DDW1] and [DDW2] in the case $\Gamma$ is any finitely generated group. We obtain:

Theorem 1.3 (The Morgan-Shalen compactification). Let $\left\{\rho_{k}\right\}_{k=1,2, \ldots}$ be a sequence of unbounded irreducible $S L(2, \mathbf{C})$ representations of $\Gamma$ and $p$ : $\bar{X} \rightarrow X$ a covering of a flat admissible 2-complex $X$ without boundary with $\Gamma$ as its transformation group. Let $u_{k}: \bar{X} \rightarrow \mathbf{H}^{3}, k=1,2, \ldots$, be a sequence of $\rho_{k}$-equivariant harmonic maps and $f_{k}: \bar{X} \rightarrow Y_{k}$ be a sequence of maps obtained by rescaling the target of $u_{k}$ so that the energy of $f_{k}$ is equal to 1 . Then there exists a subsequence of $f_{k}$ which converges (in the sense of [KS1]) to a nonconstant equivariant harmonic map $f_{\infty}: \bar{X} \rightarrow T$ where $T$ is a minimal $\mathbf{R}$-tree. The length function of the action is in the projective class of the Morgan-Shalen limit of the sequence $\left\{\rho_{k}\right\}$.

We briefly outline our approach. For Theorem 1.1, we take advantage of the fact that the domain of our harmonic map is of dimension 2. Since the regularity of harmonic maps in the interior of a face (i.e. a 2-simplex) is already known, it is enough to consider a neighborhood of an edge (i.e. a 1-simplex) point away from the vertices (i.e. 0 -simplices) of $X$. Thus, without the loss of generality, we consider a harmonic map $f$ from a union of $N$ upper half disks $D^{+}$with the $x$-axis identified to each other. We call this space $X_{1}$. Using the standard theory of harmonic maps from a surface, we know that $f$ defines a Hopf differential

$$
\left(\left|\frac{\partial f}{\partial x}\right|^{2}-\left|\frac{\partial f}{\partial y}\right|^{2}-2 i \frac{\partial f}{\partial x} \cdot \frac{\partial f}{\partial x}\right) d z^{2}
$$

on the interior of each half disk. Here, the norm squared and the dot product of the partial derivatives are interpreted in the sense of [KS1] since we allow the target space $Y$ to be non-smooth. Furthermore, it is crucial that we fix the complex structure on each half disk so that each half disk induces the same orientation on the $x$-axis that it shares with the other half disks. For convenience, we will refer to the complex function

$$
\left(\left|\frac{\partial f}{\partial x}\right|^{2}-\left|\frac{\partial f}{\partial x}\right|^{2}-2 i \frac{\partial f}{\partial x} \cdot \frac{\partial f}{\partial x}\right)
$$

as the Hopf function. 
To motivate the use of the Hopf functions in our paper, we will first examine the simple case when $N=2$ and the $\mathbf{R}$-tree is the real number line. Thus, we are considering the case of a harmonic function from the unit disk. This defines the usual Hopf function $\phi$ on the unit disk. Now label the upper half disk as $D_{1}^{+}$and the lower half disk as $D_{2}^{+}$and consider the Hopf functions $\phi_{1}$ and $\phi_{2}$ with respect to the complex structures on $D_{1}^{+}$and $D_{2}^{+}$which induces the same orientation on the $x$-axis. In particular, this means we use the complex coordinates $(x, y) \in D^{+} \mapsto(x,-y) \in D_{2}^{+}$on the lower half disk. Thus the Hopf functions are given by

$$
\phi_{1}(z)=\phi(z) \text { and } \phi_{2}(z)=\bar{\phi}(\bar{z}) \text {. }
$$

Now note that this means

$$
\operatorname{Im}\left(\phi_{1}(x, 0)+\phi_{2}(x, 0)\right)=\operatorname{Im}(\phi(x, 0)+\bar{\phi}(x, 0))=0 .
$$

We will show that in general, the imaginary part of the Hopf functions sums to 0 on the $x$-axis. Thus, by the reflection principle, the sum of the Hopf functions extends to the whole disk $D$. From this, we will show that $\left|\frac{\partial f}{\partial x}\right|^{2}$ and $\left|\frac{\partial f}{\partial y}\right|^{2}$ are bounded with bounds only dependent on the total energy of $f$ and the distance to the boundary. Thus, if $f: X \rightarrow Y$ is a harmonic map, then $f$ is Lipschitz continuous away from the vertices of $X$ with Lipschitz constant dependent only on the total energy and the distance to the vertices. Using this regularity result and some analysis near the vertices, it is not too hard to prove the global Hölder regularity of $f$ with the Hölder constant and exponent dependent only on the total energy.

The corresponding compactness result of Theorem 1.2 for smooth domains is shown in [KS1]. The main difficulty in extending their result to our situation is, apriori, there is a possibility that the energies of $f_{k}$ will concentrate at the vertices and the edges of $X_{k}$, and this can lead to the limit map being a constant map (on the fundamental domain). The Lipschitz regularity of $f_{k}$, allows us to eliminate this scenario away from the vertices. To do likewise at a vertex, we use the Hölder continuity as well as prove a monotone property of energy. More specifically, we show that $E_{k}(r)$, the energy of $f_{k}$ in a ball of radius $r$ about a vertex, essentially decays like $r^{2 \alpha}$ where $\alpha$ is the Hölder exponent. This allows us to prove the general compactness result of Theorem 1.2.

The energies of the sequence of $\rho_{k}$-equivariant harmonic maps $u_{k}$ of Theorem 1.2 go to infinity because the sequence $\rho_{k}$ is unbounded. To prove Theorem 1.3, we apply Theorem 1.2 to the rescaled maps $f_{k}$. The intuitive idea of why the target of the limit map is a tree is that a large geodesic triangle 
in a hyperbolic space looks like a tripod (three line segments joined at a common origin). If one rescales a hyperbolic space by a large constant (i.e. the energy of $f_{k}$ ), we get a tree in the limit.

We would like to end this introduction by mentioning some closely related work. Harmonic maps from polyhedral domains were first considered in [Ch]. Under suitable assumptions on the metric, the Hölder continuity of the map was obtained. Later $[\mathrm{EF}]$ proved the pointwise Hölder continuity for a general polyhedral domain. Here, pointwise Hölder continuity indicates that the Hölder constants and exponents depended on the each point of the domain. $[\mathrm{F}]$ has improved $[\mathrm{EF}]$ to show that a harmonic map is locally uniformly Hölder continuous, but did not give a dependence to the local Hölder constant and exponent. In Theorem 1.1, we obtain a local Lipschitz continuity as well as a global Hölder continuity with the Hölder constant and exponent explicitly dependent only the energy of the map.

\section{Definitions.}

\subsection{Admissible Riemannian simplicial complex.}

A simplicial complex of dimension $n$ is referred to as a $n$-complex. A connected locally finite $n$-complex is called admissible (cf. [Ch] and [EF]) if the following two conditions hold:

(i) $X$ is dimensionally homogeneous, i.e., every simplex is contained in a $n$ simplex, and

(ii) $X$ is $(n-1)$-chainable, i.e., every two $n$-simplices $A$ and $B$ can be joined by a sequence $A=F_{0}, e_{0}, F_{1}, e_{1}, \ldots, F_{k-1}, e_{k-1}, F_{k}=B$ where $F_{i}$ is a $n$-simplex and $e_{i}$ is a $(n-1)$-simplex contained in $F_{i}$ and $F_{i+1}$.

The boundary $\partial X$ of $X$ is the union of all simplices of dimension $n-1$ which is contained in only one $n$ dimensional simplex. Here and henceforth, we use the convention that simplices are closed. A locally finite simplicial complex is called a Riemannian simplicial complex if a smooth Riemannian metric is defined on each top dimensional simplex. This set of Riemannian metrics induces a distance function on $X$ which we will denote by $d_{X}(\cdot, \cdot)$.

For $p_{0} \in X$, we will denote the ball of radius $\sigma$ centered around a point $p_{0}$ by $B_{\sigma}\left(p_{0}\right)=\left\{q \in X: d_{X}\left(p_{0}, q\right)<\sigma\right\}$ and the union of the simplices that contain $p_{0}$ as $\operatorname{st}\left(p_{0}\right)$. Also let $\varrho\left(p_{0}\right)=\sup \left\{r: B_{r}\left(p_{0}\right) \subset \operatorname{st}\left(p_{0}\right)\right\}$. 


\subsection{The two dimensional case.}

For simplicity, we will refer to a two dimensional simplex as a face, a one dimensional simplex as an edge, and a zero dimensional simplex as a vertex. A point in the interior of a face (resp. an edge) is referred to as a face (resp. edge) point. We will denote the set of vertices by $V$.

Lemma 2.1. Let $X^{\prime}$ be a connected finite 2-complex. There exists an admissible finite 2-complex $X$ with the property that $\partial X=\emptyset$ and $\pi_{1}(X)=\pi_{1}\left(X^{\prime}\right)$.

Proof. We construct $X$ from $X^{(1)}=X^{\prime}$ following the steps below:

Step 1 (Dimensionally homogeneous). Let $e_{1}, e_{2}, \ldots, e_{k}$ be the edges of $X^{(1)}$ not contained in a face. For each $i$, add a new face to $X^{(1)}$ and identify one of its edges to $e_{i}$. Call the new 2-complex $X^{(2)}$. By construction, $X^{(2)}$ is a dimensionally homogeneous and $\pi_{1}\left(X^{(2)}\right)=\pi_{1}\left(X^{(1)}\right)=\pi_{1}\left(X^{\prime}\right)$.

Step $2((n-1)$-chainable). Let $V$ be the set of vertices $v$ with the property that $\operatorname{st}(v)-\{v\}$ has more than one components. Let $v_{1}, v_{2}, \ldots, v_{l}$ be the elements of $V$ and for each $i$, let $e_{i, 1}$ and $e_{i, 2}$ be two of the edges of $X^{(2)}$ containing $v_{i}$, add a new face to $X^{(2)}$ and identify two edges of the new face to $e_{i, 1}$ and $e_{i, 2}$ respectively. Call the new 2 -complex $X^{(3)}$. Note that if $v$ is any vertex on a new face, then $\operatorname{st}(v)-\{v\}$ is connected since $e_{i, 1}$ and $e_{i, 2}$ is contained in two different faces of $X^{(3)}$. Thus, $X^{(3)}$ is admissible and $\pi\left(X^{(3)}\right)=\pi_{1}\left(X^{(2)}\right)=$ $\pi_{1}\left(X^{(1)}\right)=\pi_{1}\left(X^{\prime}\right)$.

Step 3 (Without boundary). Let $e_{1}, e_{2}, \ldots, e_{m}$ be edges of $X^{(3)}$ that is contained in only one face of $X$. For each $i$, add a 2-skeleton of a 3 -simplex to $X^{(3)}$ and identify an edge of the 2-skeleton to $e_{i}$. Call this new 2-complex $X$. By construction, $X$ is admissible, without boundary and $\pi_{1}(X)=\pi_{1}\left(X^{(3)}\right)=$ $\pi_{1}\left(X^{(2)}\right)=\pi_{1}\left(X^{(1)}\right)=\pi_{1}\left(X^{\prime}\right)$.

Let $\triangle$ be an equilateral triangle with coordinates given by, say,

$$
\triangle=\left\{(x, y) \in \mathbf{R}^{2}: y \leq \sqrt{3} x+\sqrt{3}, y \leq-\sqrt{3} x+\sqrt{3}, y \geq 0\right\}
$$

and $g_{0}=d x^{2}+d y^{2}$. We will say that a two dimensional Riemannian simplicial complex $X$ is flat if, for each face $F$ of $X$ and metric $g_{F}$ on $F,\left(F, g_{F}\right)$ is isometric to $\left(\triangle, g_{0}\right)$ and if $F_{1}$ and $F_{2}$ are adjacent faces sharing an edge $e$, the metrics $g_{F_{1}}$ and $g_{F_{2}}$ induce the same distance function on $e$.

If $X$ is a flat admissible 2-complex, we can model $B_{r}\left(p_{0}\right), r<\varrho\left(p_{0}\right)=$ $\sup \left\{r: B_{r}\left(p_{0}\right) \subset \operatorname{st}\left(p_{0}\right)\right\}$, by the three cases below: 
(i) If $p_{0}$ is a face point, we isometrically identify $B_{r}\left(p_{0}\right)$ with a disk $D_{r}$ of radius $r$ centered at the origin in $\mathbf{R}^{2}$ and let $z(p)=(x, y)$ be this identification.

(ii) If $p_{0}$ is an edge point, we isometrically identify $B_{r}\left(p_{0}\right)$ with $X_{r}$ defined as follows: Let $n$ be the number of faces contained in $\operatorname{st}\left(p_{0}\right)$ and take $n$ copies of the upper half disk $D_{r}^{+}=\left\{(x, y) \in \mathbf{R}^{2} \mid x^{2}+y^{2}<r, y \geq 0\right\}$. We would like to distinguish these copies so we label them $D_{r, 1}^{+}, \ldots, D_{r, n}^{+}$and use $\left(x_{i}, y_{i}\right)$ to denote the point corresponding to $(x, y) \in D_{r}^{+}$on the $i$ th copy $D_{r, i}^{+}$. Let $X_{r}=\cup_{i=1}^{n} D_{r, i}^{+} / \sim$ where $\sim$ is defined by

$$
\left(x_{i}, 0\right) \sim\left(x_{j}, 0\right) \text { for } x \in \mathbf{R} .
$$

In other words, $\sim$ identifies the $x$-axis of $D_{r, i}^{+}$to the $x$-axis of $D_{r, j}^{+}$for all $i$ and $j$ so that $B_{r}(\sigma)$ is isometric to $X_{r}$. Let $z(p)=\left(x_{i}, y_{i}\right)$ be this identification. We will refer to $X_{r}$ as an edge piece.

(iii) If $p_{0}$ is a vertex, we isometrically identify $B_{r}\left(p_{0}\right)$ with $X_{r}^{\prime}$ defined as follows: Let $W_{r}=\left\{(x, y) \in \mathbf{R}^{2}: x^{2}+y^{2} \leq r, 0 \leq \tan ^{-1}\left(\frac{y}{x}\right) \leq \frac{\pi}{3}, x, y \geq 0\right\}$. Let $n$ be the number of faces contained in st $\left(p_{0}\right)$, take $n$ copies of $W_{r}$, label them $W_{r, 1}, \ldots, W_{n, r}$ to distinguish these copies and use $\left(x_{i}, y_{i}\right)$ to denote the point corresponding to $(x, y) \in W_{r}$ on the $i$ th copy $W_{r, i}$. Let $X_{r}^{\prime}=\cup_{i=1}^{n} W_{i} / \sim$ where $\sim$ is an equivalence relations defined so that $X_{r}^{\prime}$ is isometric to $B_{r}\left(p_{0}\right)$. Let $z(p)=\left(x_{i}, y_{i}\right)$ be this identification. We will refer to $X_{r}^{\prime}$ as a vertex piece.

\subsection{Metric spaces of curvature bounded from above.}

A complete metric space $(Y, d)$ is said to have curvature bounded from above by $\kappa$ if the following conditions are satisfied:

(i) The space $(Y, d)$ is a length space. That is, for any two points $P$ and $Q$ in $Y$, there exists a rectifiable curve $\gamma_{P Q}$ so that the length of $\gamma_{P Q}$ is equal to $d(P, Q)$ (which we will sometimes denote by $d_{P Q}$ for simplicity). We call such distance realizing curves geodesics.

(ii) Let $a=\sqrt{|\kappa|}$. Every point $P_{0} \in Y$ has a neighborhood $U \subset Y$ so that given $P, Q, R \in U$ (assume $d_{P Q}+d_{Q R}+d_{R P}<\frac{\pi}{\sqrt{\kappa}}$ for $\kappa>0$ ) with $Q_{t}$ defined to be the point on the geodesic $\gamma_{Q R}$ satisfying $d_{Q Q_{t}}=t d_{Q R}$ and $d_{Q t}=(1-t) d_{Q R}$, we have

$$
\cosh \left(a d_{P Q_{t}}\right) \leq \frac{\sinh \left((1-t) a d_{Q R}\right)}{\sinh \left(a d_{Q R}\right)} \cosh a d_{P Q}+\frac{\sinh \left(\operatorname{tad}_{Q R}\right)}{\sinh \left(a d_{Q R}\right)} \cosh a d_{P R}
$$

for $\kappa<0$,

$$
d_{P Q_{t}}^{2} \leq(1-t) d_{P Q}^{2}+t d_{P R}^{2}-t(1-t) d_{Q R}^{2}
$$


for $\kappa=0$, and

$$
\cos \left(a d_{P Q_{t}}\right) \geq \frac{\sin \left((1-t) a d_{Q R}\right)}{\sin \left(a d_{Q R}\right)} \cos a d_{P Q}+\frac{\sin \left(\operatorname{tad}_{Q R}\right)}{\sin \left(a d_{Q R}\right)} \cos a d_{P R}
$$

for $\kappa>0$.

We will say that $Y$ is NPC (non-positively curved) if it has curvature bounded from above by 0 . A simply connected space of curvature bounded from above by $\kappa<0$ is commonly referred to as a $C A T(\kappa)$ space in literature. If a metric space $(T, d)$ is a $C A T(\kappa)$ space for any $\kappa$, then $(T, d)$ a $C A T(\infty)$ space.

A R-tree is a metric space $(T, d)$ so that for any $t, s \in T$, there exists a path $\gamma:[a, b] \rightarrow T$ joining $t$ to $s$ so that length $(\gamma)=d(t, s)$ and $\gamma$ is the unique embedded path from $x$ to $y$. Here,

$$
\operatorname{length}(\gamma)=\inf \sum_{i=1}^{n} d\left(\gamma\left(\tau_{i-1}\right), \gamma\left(\tau_{i}\right)\right)
$$

where inf is taken over all partitions $a=\tau_{0}<\tau_{1}<\ldots<\tau_{n-1}<\tau_{n}=b$ of $[a, b]$.

It is known that $(T, d)$ is a $\mathbf{R}$-tree if and only if $(T, d)$ is a $\operatorname{CAT}(\infty)$ space.

\subsection{Harmonic maps.}

A map from $X$ into $Y$ is called harmonic if it is locally energy minimizing. Recall that, when $\left(X^{m}, g\right)$ and $\left(Y^{n}, h\right)$ are Riemannian manifolds, then the energy of $f: X \rightarrow Y$ is

$$
E^{f}:=\int_{X}|\nabla f|^{2} d \mu
$$

where

$$
|\nabla f|^{2}(x)=\sum_{\alpha, \beta=1}^{m} g^{\alpha \beta}(x) h_{i j}(f(x)) \frac{\partial f^{i}}{\partial x^{\alpha}} \frac{\partial f^{j}}{\partial x^{\beta}}
$$

with $\left(x^{\alpha}\right)$ and $\left(f^{i}\right)$ the local coordinate systems around $x \in X$ and $f(x) \in Y$ respectively.

If $X$ is a Riemannian manifold but $Y$ is only assumed to be a metric space, then we use the Korevaar-Schoen definition of energy: $E^{f}$ is defined as above with $|\nabla f|^{2} d \mu$ the weak limit of $\epsilon$-approximate energy density measures which are measures derived from the appropriate average difference quotients. More specifically, define $e_{\epsilon}: X \rightarrow \mathbf{R}$ by

$$
e_{\epsilon}(x)=\left\{\begin{array}{cc}
\int_{y \in S(x, \epsilon)} \frac{d^{2}(f(x), f(y))}{\epsilon^{2}} \frac{d \sigma_{x, \epsilon}}{\epsilon^{n-1}} & \text { for } x \in X_{\epsilon} \\
0 & \text { for } x \in X-X_{\epsilon}
\end{array}\right.
$$


where $\sigma_{x, \epsilon}$ is the induced measure on the $\epsilon$-sphere $S(x, \epsilon)$ centered at $x$ and $X_{\epsilon}=\{x \in X: d(x, \partial X)>\epsilon\}$. We define a family of functionals $E_{\epsilon}^{f}$ : $C_{c}(X) \rightarrow \mathbf{R}$ by setting

$$
E_{\epsilon}^{f}(\varphi)=\int_{X} \varphi e_{\epsilon} d \mu
$$

We say $f$ has finite energy (or that $f \in W^{1,2}(X, Y)$ ) if

$$
E^{f}:=\sup _{\varphi \in C_{c}(X), 0 \leq \varphi \leq 1} \limsup _{\epsilon \rightarrow 0} E_{\epsilon}^{f}(\varphi)<\infty .
$$

It can be shown that if $f$ has finite energy, the measures $e_{\epsilon}(x) d x$ converge weakly to a measure which is absolutely continuous with respect to the Lebesgue measure. Therefore, there exists a function $e(x)$, which we call the energy density, so that $e_{\epsilon}(x) d \mu \rightarrow e(x) d \mu$. In analogy to the case of real valued functions, we write $|\nabla f|^{2}(x)$ in place of $e(x)$. In particular,

$$
E^{f}=\int_{X}|\nabla f|^{2} d \mu
$$

For $V \in \Gamma X$ where $\Gamma X$ is the set of Lipschitz vector fields on $X,\left|f_{*}(V)\right|^{2}$ is similarly defined. The real valued $L^{1}$ function $\left|f_{*}(V)\right|^{2}$ generalizes the norm squared on the directional derivative of $f$. The generalization of the pull-back metric is

$$
\pi_{f}(V, W)=\Gamma X \times \Gamma X \rightarrow L^{1}(X, \mathbf{R})
$$

where

$$
\pi_{f}(V, W)=\frac{1}{2}\left|f_{*}(V+W)\right|^{2}-\frac{1}{2}\left|f_{*}(V-W)\right|^{2} .
$$

We refer to $[\mathrm{KS} 1]$ for more details.

Finally, the Korevaar-Schoen definition of energy (cf. [Ch] and [EF]) can be extended to the case when $X$ is an admissible Riemannian simplicial complex. Here, the energy $E^{f}$ is

$$
\int_{X}|\nabla f|^{2} d \mu:=\int_{\cup_{i=1}^{k} F_{i}}|\nabla f|^{2} d \mu
$$

where $\left\{F_{i}\right\}_{k=1, \ldots, k}$ are the top-dimensional simplices of $X$. The functions $|\nabla f|^{2}$ and $\left|f_{*}(V)\right|^{2}$ are defined for almost every point in $X$.

For a flat admissible 2-complex $X$ and coordinate functions $x, y$ defined on a face of $X$, we will write $\left|\frac{\partial f}{\partial x}\right|^{2}$ to denote $\left|f_{*}\left(\frac{\partial}{\partial x}\right)\right|^{2}$ and $\frac{\partial f}{\partial x} \cdot \frac{\partial f}{\partial y}$ to denote $\pi_{f}\left(\frac{\partial}{\partial x}, \frac{\partial}{\partial y}\right)$. 


\section{Regularity results.}

We derive some regularity results for a harmonic map $f: X \rightarrow Y$ assuming that $X$ is a flat admissible finite 2-complex without boundary and that $Y$ is a NPC space as defined in Section 2. We start by proving a series of propositions which are the singular analogues of results in [GS]. These results are crucial in our arguments later. The following is a restatement of Proposition 2.2 in [GS].

Proposition 3.1. Let $X$ and $Y$ as above and $Q \in Y$. If $f: X \rightarrow Y$ is a harmonic map, then $\triangle d^{2}(f(x), Q)-2|\nabla f|^{2} \geq 0$ weakly, i.e.

$$
-\int_{X} \nabla d^{2}(f(x), Q) \cdot \nabla \varphi \geq 2 \int_{X}|\nabla f|^{2} \varphi
$$

for any $\varphi \in W_{c}^{1,2}(X, \mathbf{R})$.

Proof. The computation for the proof of this inequality can be found in the proof of [EF] Lemma 10.2 which is based on the proof of [GS] Proposition 2.2.

Choosing $\varphi$ to approximate the characteristic function of a small geodesic ball $B_{\sigma}\left(p_{0}\right)$, we get

$$
2 \int_{B_{\sigma}\left(p_{0}\right)}|\nabla f|^{2} d \mu \leq \int_{\partial B_{\sigma}\left(p_{0}\right)} \frac{\partial}{\partial r} d^{2}(f, Q) d s .
$$

Proposition 3.2. Let $X$ and $Y$ as above and $f: X \rightarrow Y$ be a harmonic map. For $p_{0} \in X$, define

$$
E(r)=\int_{B_{r}\left(p_{0}\right)}|\nabla f|^{2} d \mu
$$

and

$$
I(r)=\int_{\partial B_{r}\left(p_{0}\right)} d^{2}\left(f, f\left(p_{0}\right)\right) d s
$$

Then

$$
r \mapsto \operatorname{Ord}(p, r)=\frac{r E(r)}{I(r)}
$$

is a non-decreasing function for $r<\varrho\left(p_{0}\right)=\sup \left\{r: B_{r}\left(p_{0}\right) \subset \operatorname{st}\left(p_{0}\right)\right\}$ and hence

$$
\alpha=\lim _{r \rightarrow 0} \frac{r E(r)}{I(r)}
$$

exists. 
Remark 3.3. The difficulty in working with harmonic maps from a simplicial complex is that the monotonicity property for $r \mapsto \frac{r E(r)}{I(r)}$ does not necessarily hold for $r>\varrho$. We illustrate this by the following example. Let $X_{1}$ be the space consisting of three upper half disks $D_{1}^{+}, D_{2}^{+}$, and $D_{3}^{+}$identified along the $x$-axis and consider the harmonic function $f: X_{1} \rightarrow \mathbf{R}$ with $f_{j}=\left.f\right|_{D_{j}^{+}}$ defined by $f_{1}(x, y)=y, f_{2}(x, y)=y$ and $f_{3}(x, y)=0$. Fix $p_{0}=\left(x_{0}, y_{0}\right) \in$ $D_{3}^{+} \subset X_{1}$. Now identify $D_{j}^{+}$(for $j=1,2$ ) and $D_{3}^{+}$to the upper and lower half disk of the unit disk $D$ respectively by the embedding defined by $(x, y) \in$ $D_{j}^{+} \mapsto(x, y) \in D$ and $(x, y) \in D_{3}^{+} \mapsto(x,-y) \in D$. We let $(r, \gamma)$ be the polar coordinates of $D$ centered at $\left(x_{0},-y_{0}\right)$. (Note that $\left(x,-y_{0}\right)$ is the image of $p_{0}=\left(x_{0}, y_{0}\right) \in D_{j}^{+}$under the embedding.) The set $\partial B_{r}\left(p_{0}\right) \cap D_{j}^{+}(j=1$ or 2) can be parameterized by the angular coordinate $\gamma$. More specifically, if we let $\theta=\cos ^{-1}\left(\frac{y_{0}}{r}\right)$, then $\gamma \mapsto(r, \gamma)$ for $-\theta \leq \gamma \leq \theta$ gives us the portion on $\partial B_{r}\left(p_{0}\right)$ in $D_{j}^{+}$. For $(x, y) \in \partial B_{r}\left(p_{0}\right) \cap D_{j}^{+}(j=1,2), y$ is given in terms of $\gamma$ by

$$
y=r \cos \gamma-r \cos \theta
$$

by elementary geometry. Since $f$ is identically constant on $D_{3}^{+}$, we have

$$
\begin{aligned}
d^{2}\left(f(x, y), f\left(x_{0}, y_{0}\right)\right) & =y^{2} \\
& =(r \cos \gamma-r \cos \theta)^{2} \\
& =r^{2}\left(\cos ^{2} \gamma-2 \cos \gamma \cos \theta+\cos ^{2} \theta\right) \\
& =r^{2}\left(\frac{1}{2}+\frac{\cos 2 \gamma}{2}-2 \cos \gamma \cos \theta+\cos ^{2} \theta\right) .
\end{aligned}
$$

Therefore,

$$
\begin{aligned}
I(r) & =\int_{\partial B_{r}\left(p_{0}\right)} d^{2}\left(f(x, y), f\left(x_{0}, y_{0}\right)\right) d s \\
& =\sum_{j=1}^{2} \int_{\partial B_{r}\left(p_{0}\right) \cap D_{j}^{+}} d^{2}\left(f(x, y), f\left(x_{0}, y_{0}\right)\right) d s \\
& =2 r^{3} \int_{-\theta}^{\theta}\left(\frac{1}{2}+\frac{\cos 2 \gamma}{2}-2 \cos \gamma \cos \theta+\cos ^{2} \theta\right) d \gamma \\
& =2 r^{3}\left(2 \theta-\frac{3}{2} \sin 2 \theta+(\cos 2 \theta) \theta\right) .
\end{aligned}
$$


Furthermore, $|\nabla f|^{2}=1$ in $D_{1}^{+}$and $D_{2}^{+}$and $|\nabla f|^{2}=0$ in $D_{3}^{+}$, so

$$
\begin{aligned}
E(r) & =\int_{B_{r}\left(p_{0}\right)}|\nabla f|^{2} \\
& =\sum_{j=1}^{2} \int_{B_{r}\left(p_{0}\right) \cap D_{j}^{+}}|\nabla f|^{2} \\
& =\operatorname{Area}\left(B_{r}\left(p_{0}\right) \cap D_{j}^{+}\right) \\
& =2 r^{2}\left(\theta-\frac{\sin 2 \theta}{2}\right) .
\end{aligned}
$$

Now note that

$$
\lim _{r \rightarrow y_{0}^{+}} \frac{r E(r)}{I(r)}=\lim _{\theta \rightarrow 0^{+}} \frac{2 r^{3}\left(\theta-\frac{\sin 2 \theta}{2}\right)}{2 r^{3}\left(2 \theta-\frac{3}{2} \sin 2 \theta+(\cos 2 \theta) \theta\right)}=\infty
$$

and this shows that $r \mapsto \frac{r E(r)}{I(r)}$ is not a non-decreasing function for $r>\varrho\left(p_{0}\right)$.

Proof. (Proposition 3.2) Let $\zeta: X \rightarrow \mathbf{R}_{+} \cup\{0\}$ be a continuous function which is smooth on each face of $X$. For $p_{0} \in X, r<\varrho\left(p_{0}\right)$ and any $p \in B_{r}\left(p_{0}\right)$, a geodesic from $p_{0}$ to $p$ can be uniquely extended beyond $p$ to a geodesic of length $r$. Thus, for sufficiently small $t$ so that $(1+t \zeta(p)) d_{X}\left(p, p_{0}\right)<\varrho\left(p_{0}\right)$, we can define $(1+t \zeta(p)) p$ as the point on this extension that is distance $(1+$ $t \zeta(p)) d_{X}\left(p, p_{0}\right)$ away from $p_{0}$. For $\zeta$ with $\operatorname{spt}(\zeta) \subset B_{r}\left(p_{0}\right)$ and $t$ sufficiently small, we define $F_{t}: X \rightarrow X$ as

$$
F_{t}(p)=\left\{\begin{array}{cc}
(1+t \zeta(p)) p & \text { for } p \in B_{r}\left(p_{0}\right) \\
\text { identity } & \text { for } p \in X-B_{r}\left(p_{0}\right)
\end{array}\right.
$$

With that, we can now follow the usual calculation to prove Proposition 3.2. In other words, the standard computation (see [GS], Section 2 for example) done on each face of $X$ gives

$$
0=\int_{B_{r}\left(p_{0}\right)} \frac{\partial \zeta}{\partial x} x\left(\frac{\partial f}{\partial x}\right)^{2}+\frac{\partial \zeta}{\partial y} y\left(\frac{\partial f}{\partial y}\right)^{2}+2 \frac{\partial \zeta}{\partial x} y \frac{\partial f}{\partial x} \frac{\partial f}{\partial y}+2 \frac{\partial \zeta}{\partial y} x \frac{\partial f}{\partial x} \frac{\partial f}{\partial y}
$$

Taking $\zeta$ to be an approximation of the characteristic function of the ball $B_{r}\left(p_{0}\right)$, we get

$$
E^{\prime}(r)=2 \int_{\partial B_{r}\left(p_{0}\right)}\left|\frac{\partial f}{\partial r}\right|^{2} d s
$$


for a.e. $r<\varrho\left(p_{0}\right)=\sup \left\{r: B_{r}\left(p_{0}\right) \subset \operatorname{st}\left(p_{0}\right)\right\}$. Again, for a.e. $r<\varrho\left(p_{0}\right)$, standard computation on each face of $X$ gives,

$$
I^{\prime}(r)=\int_{\partial B_{r}\left(p_{0}\right)} \frac{\partial}{\partial r} d^{2}\left(f, f\left(p_{0}\right)\right) d s+\frac{I(r)}{r} .
$$

The above two equations along with (3.1), the Schwarz inequality,

$$
\begin{aligned}
& \left.\left(\int_{B_{r}\left(p_{0}\right)} d\left(f, f\left(p_{0}\right)\right) \frac{\partial}{\partial r} d(f, f(0))\right) d s\right)^{2} \\
& \leq\left(\int_{B_{r}\left(p_{0}\right)} d^{2}\left(f, f\left(p_{0}\right)\right) d s\right)\left(\int_{B_{r}\left(p_{0}\right)}\left(\frac{\partial}{\partial r} d(f, f(0))\right)^{2} d s\right)
\end{aligned}
$$

and the inequality,

$$
\frac{\partial}{\partial r} d\left(f, f\left(p_{0}\right)\right) \leq\left|\frac{\partial f}{\partial r}\right|
$$

imply

$$
\begin{aligned}
& \frac{d}{d r} \log \left(\frac{I(r)}{r E(r)}\right) \\
&=\frac{I^{\prime}(r)}{I(r)}-\frac{1}{r}-\frac{E^{\prime}(r)}{E(r)} \\
& \leq \quad(E(r) I(r))^{-1}\left[E(r) \int_{\partial B_{r}\left(p_{0}\right)} \frac{\partial}{\partial r}\left(d^{2}\left(f, f\left(p_{0}\right)\right)\right) d s\right. \\
&\left.\quad-2 I(r)\left(\int_{\partial B_{r}\left(p_{0}\right)}\left|\frac{\partial f}{\partial r}\right|^{2} d s\right)\right] \\
& \leq \quad 2(E(r) I(r))^{-1}\left[\left(\int_{\partial B_{r}\left(p_{0}\right)} d\left(f, f\left(p_{0}\right)\right) \frac{\partial}{\partial r} d(f, f(0)) d s\right)^{2}\right. \\
&\left.\quad-\left(\int_{\partial B_{r}\left(p_{0}\right)} d^{2}\left(f, f\left(p_{0}\right)\right) d s\right)\left(\int_{\partial B_{r}\left(p_{0}\right)}\left|\frac{\partial f}{\partial r}\right|^{2} d s\right)\right] \\
& \leq 0 .
\end{aligned}
$$

for a.e. $r<\varrho\left(p_{0}\right)$.

Remark 3.4. For a general Riemannian simplicial complex $X$, the variation $F_{t}$ defined in the proof of Proposition 3.2 does not make sense. Specifically, we 
must be able to uniquely extend a geodesic from $p$ to $p_{0}$ to a geodesic emanating from point $p_{0}$ of length $(1+t \zeta(p)) d\left(p_{0}, p\right)$. Therefore, a necessary condition to define $F_{t}$ is a positive injectivity radius at $p_{0}$. A Riemannian simplicial complex does not have this condition generally. For example, take two copies of the (flat) unit disk and identify the upper half of the unit circle of each disk to another. Then any two points of the upper half circle can be connected by two geodesics, one contained in each copy of the disk. Thus, the injectivity radius of those points is 0 .

Corollary 3.5. If

$$
\alpha_{0}=\lim _{r \rightarrow 0} \frac{r E(r)}{I(r)} \text { and } \alpha_{r}=\frac{r E(r)}{I(r)} \text { for } 0<r<\varrho\left(p_{0}\right),
$$

then

$$
\left.\frac{d}{d \sigma}\left(\frac{I(\sigma)}{\sigma^{2 \alpha_{r}+1}}\right)\right|_{\sigma=s} \geq 0
$$

and

$$
\left.\frac{d}{d \sigma}\left(\frac{E(\sigma)}{\sigma^{2 \alpha_{r}}}\right)\right|_{\sigma=s} \geq 0
$$

for $r \leq s<\varrho\left(p_{0}\right)$

Proof. Proposition 3.2 implies that

$$
\alpha_{r} I(\sigma) \leq \sigma E(\sigma) \text { for } r \leq \sigma<\varrho\left(p_{0}\right) .
$$

Furthermore, inequality (3.1) implies that

$$
2 E(\sigma) \leq \int_{\partial B_{\sigma}\left(p_{0}\right)} \frac{\partial}{\partial r} d^{2}(f, f(v)) d s=I^{\prime}(\sigma)-\frac{1}{\sigma} I(\sigma) .
$$

Combining the two inequalities,

$$
\alpha_{r} I(\sigma) \leq \frac{1}{2}\left(\sigma I^{\prime}(\sigma)-I(\sigma)\right)
$$

which implies

$$
\frac{2 \alpha_{r}+1}{\sigma} \leq \frac{I^{\prime}(\sigma)}{I(\sigma)}
$$

and hence

$$
0 \leq\left(\frac{I(\sigma)}{\sigma^{2 \alpha_{r}+1}}\right)^{\prime} \text { for } r<\sigma<\varrho\left(p_{0}\right)
$$


Again, by Proposition 3.2,

$$
\frac{2 \alpha+1}{\sigma} \leq \frac{I^{\prime}(\sigma)}{I(\sigma)} \leq \frac{1}{\sigma}+\frac{E^{\prime}(\sigma)}{E(\sigma)}
$$

which implies

$$
0 \leq\left(\frac{E(\sigma)}{\sigma^{2 \alpha_{r}}}\right)^{\prime} \text { for } r<\sigma<\varrho\left(p_{0}\right)
$$

Let $p$ be an edge point or a vertex with $s t(p)$ containing $n$ faces and let $\sigma<\varrho(p)=\sup \left\{r: B_{r}\left(p_{0}\right) \subset \operatorname{st}\left(p_{0}\right)\right\}$. Then $\partial B_{\sigma}(p)$ can be considered a graph with $n$ edges where, for each face $F, \partial B_{\sigma}(p) \cap F$ is an edge and, for each edge $e, \partial B_{\sigma}(p) \cap e$ are two vertices. The metric on each edge is inherited from the metric on the face of $X$ containing that edge. Thus, each edge has length $\pi \sigma$ if $p$ is an edge point or $\frac{\pi \sigma}{3}$ if $p$ is a vertex. Let $d s$ be the corresponding volume form on the edges and let $\bar{f}_{\sigma} \in Y$ be the point in $Y$ so that

$$
\int_{\partial B_{\sigma}(p)} d^{2}\left(f, \bar{f}_{\sigma}\right) d s=\inf _{Q \in Y} \int_{\partial B_{\sigma}(p)} d^{2}(f, Q) d s .
$$

The existence of $\bar{f}_{\sigma}$ is guaranteed by Lemma 2.5.1 of [KS1].

Proposition 3.6. There exists a constant c depending only on $n$ so that

$$
\int_{\partial B_{\sigma}(p)} d^{2}\left(f, \bar{f}_{\sigma}\right) d s \leq c \sigma^{2} \int_{\partial B_{\sigma}(p)}\left|\frac{\partial f}{\partial s}\right|^{2} d s .
$$

Proof. Assume $p$ is a vertex. The case when $p$ is an edge point follows by an analogous argument. The universal covering space of $\partial B_{\sigma}(p)$ is a simplicial tree $\tilde{T}$ and we denote a fundamental domain in this universal cover space by $T$. The finite tree $T$ has $n$ edges and its diameter $D$ is $\leq \frac{\pi \sigma n}{3}$. Let $\tilde{f}: \tilde{T} \rightarrow Y$ be the lift of $\left.f\right|_{\partial B_{\sigma}(p)}$ to the universal cover.

For $p_{0}, p_{1} \in T$, let $t \mapsto p_{t}$ for $t \in[0,1]$ be the constant speed parametrization of the unique geodesic between $p_{0}$ and $p_{1}$. The tangent vector $\dot{p}_{t}$ has length $\leq D$. For a.e. $\left(p_{0}, p_{1}\right) \in T \times T$, we have

$$
\begin{aligned}
d^{2}\left(\tilde{f}\left(p_{0}\right), \tilde{f}\left(p_{1}\right)\right) & \leq \int_{0}^{1}\left|\tilde{f}_{*}\left(\dot{p}_{t}\right)\right|^{2} d t \\
& \leq D^{2} \int_{0}^{1}\left|\frac{\partial \tilde{f}}{\partial s}\right|^{2}\left(p_{t}\right) d t
\end{aligned}
$$


For a fixed $p_{0}$ and $q \in T$, there are at most $n$ ! points $p_{1}$ so that $d\left(p_{0}, q\right)=t$ and $d\left(q, p_{1}\right)=1-t$. It then follows that

$$
\int_{T}\left|\frac{\partial \tilde{f}}{\partial s}\right|^{2}\left(p_{t}\right) d s\left(p_{1}\right) \leq n ! \int_{T}\left|\frac{\partial \tilde{f}}{\partial s}\right|^{2}(q) d s(q)
$$

and

$$
\begin{aligned}
\int_{T} d^{2}\left(\tilde{f}\left(p_{1}\right), \bar{f}_{\sigma}\right) d s\left(p_{1}\right) & \leq \int_{T} d^{2}\left(\tilde{f}\left(p_{0}\right), \tilde{f}\left(p_{1}\right)\right) d s\left(p_{1}\right) \\
& \leq D^{2} \int_{T} \int_{0}^{1}\left|\frac{\partial \tilde{f}}{\partial s}\right|^{2}\left(p_{t}\right) d t d s\left(p_{1}\right) \\
& =D^{2} \int_{0}^{1} \int_{T}\left|\frac{\partial \tilde{f}}{\partial s}\right|^{2}\left(p_{t}\right) d s\left(p_{1}\right) d t \\
& \leq D^{2} n ! \int_{0}^{1} \int_{T}\left|\frac{\partial \tilde{f}}{\partial s}\right|^{2}(q) d s(q) d t \\
& =\frac{\pi^{2} n^{2} n ! \sigma^{2}}{3^{2}} \int_{T}\left|\frac{\partial \tilde{f}}{\partial s}\right|^{2} d s
\end{aligned}
$$

and we have shown inequality (3.7).

The local Hölder continuity for a harmonic map $f$ from an admissible Riemannian simplicial complex to a NPC space has been claimed in [Ch] and [EF]. We note the proof of $[\mathrm{Ch}]$ is disputed in $[\mathrm{EF}]$. On the other hand, the idea of $[\mathrm{Ch}]$ is valid as long as the following additional assumption is imposed: for every $p \in X$ and $q \in B_{r_{0}}(p)$ where $r_{0}=\varrho(p)$, the geodesic between $p$ and $q$ can be uniquely extended to a geodesic of length $r_{0}$. This is certainly true for $X$ as above.

Our arguments above immediately yield a proof of the local Hölder continuity which we present for the sake of completeness.

Theorem 3.7. Let $X$ and $Y$ as above. If $f: X \rightarrow Y$ is a harmonic map, then $f$ is locally Hölder continuous. More specifically, for every $p_{0} \in X$, there exist constants $A$ and $\alpha$ depending only on $r_{0}=\varrho(p), E^{f}$ and $n$, the number of faces intersection $B_{r_{0}}\left(p_{0}\right)$, so that

$$
d(f(p), f(q)) \leq A d_{X}^{\alpha}(p, q) \text { for all } p, q \in B_{r_{0} / 2}(p) .
$$


Proof. Let $p \in B_{r_{0} / 2}\left(p_{0}\right)$ and $E(\sigma)=\int_{B_{\sigma}(p)}|\nabla f|^{2} d \mu$ for $\sigma \leq r_{0}$. By inequality (3.1) and the fact that $\frac{\partial}{\partial r} d\left(f, \bar{f}_{\sigma}\right) \leq\left|\frac{\partial f}{\partial r}\right|$, we have

$$
\begin{aligned}
E(\sigma) & \leq \frac{1}{2} \int_{\partial B_{\sigma}(p)} \frac{\partial}{\partial r} d^{2}\left(f, \bar{f}_{\sigma}\right) d s \\
& =\int_{\partial B_{\sigma}(p)} d\left(f, \bar{f}_{\sigma}\right) \frac{\partial}{\partial r} d\left(f, \bar{f}_{\sigma}\right) d s \\
& \leq \int_{\partial B_{\sigma}(p)} d\left(f, \bar{f}_{\sigma}\right)\left|\frac{\partial f}{\partial r}\right| d s \\
& \leq\left(\int_{\partial B_{\sigma}(p)} d^{2}\left(f, \bar{f}_{\sigma}\right) d s\right)^{1 / 2}\left(\int_{\partial B_{\sigma}(p)}\left|\frac{\partial f}{\partial r}\right|^{2} d s\right)^{1 / 2}
\end{aligned}
$$

Thus, by inequality (3.7),

$$
E(\sigma) \leq \sqrt{c} \sigma\left(\int_{\partial B_{\sigma}(p)}\left|\frac{\partial f}{\partial s}\right|^{2} d s\right)^{1 / 2}\left(\int_{\partial B_{\sigma}(p)}\left|\frac{\partial f}{\partial r}\right|^{2} d s\right)^{1 / 2}
$$

By equality (3.3),

$$
\begin{aligned}
\int_{\partial B_{\sigma}(p)}\left|\frac{\partial f}{\partial s}\right|^{2} d s & =\int_{\partial B_{\sigma}(p)}|\nabla f|^{2} d s-\int_{\partial B_{\sigma}(p)}\left|\frac{\partial f}{\partial r}\right|^{2} d s \\
& =E^{\prime}(\sigma)-\int_{\partial B_{\sigma}(p)}\left|\frac{\partial f}{\partial r}\right|^{2} d s \\
& =\int_{\partial B_{\sigma}(p)}\left|\frac{\partial f}{\partial r}\right|^{2} d s .
\end{aligned}
$$

Combining (3.8) and (3.9), we obtain

$$
E(\sigma) \leq \sqrt{c} \sigma \int_{\partial B_{\sigma}(p)}\left|\frac{\partial f}{\partial r}\right|^{2} d s \leq \sqrt{c} \sigma E^{\prime}(\sigma)
$$

or

$$
\frac{2 \alpha}{\sigma} \leq \frac{E^{\prime}(\sigma)}{E(\sigma)}
$$

where $2 \alpha=\frac{1}{\sqrt{c}}$. Integrating this inequality from $r$ to $r_{0}$, we have

$$
E(r) \leq \frac{E\left(r_{0}\right)}{r_{0}^{2 \alpha}} r^{2 \alpha} \leq \frac{E^{f}}{r_{0}^{2 \alpha}} r^{2 \alpha}
$$


Now we apply Morrey's energy decay argument to prove Hölder continuity. Let $p, q \in B_{r_{0} / 2}(p)$ with $d(p, q)=r$ and let $\bar{p}_{t}, 0 \leq t \leq 1$, be the point which is fraction $t$ along the geodesic from $p$ to $q$. For any $p^{\prime} \in B_{\frac{r}{2}}\left(\bar{p}_{\frac{1}{2}}\right)$, let $p_{t}, 0 \leq t \leq 1$, be an arclength parameterized geodesic from $p$ to $p^{\prime}$. Since $d\left(p, p^{\prime}\right) \leq d\left(p, \bar{p}_{\frac{1}{2}}\right)+$ $d\left(\bar{p}_{\frac{1}{2}}, p^{\prime}\right) \leq r$, we have that the tangent vector $\dot{p}_{t}$ has length $\leq r$. Therefore,

$$
\begin{aligned}
d\left(f(p), f\left(p^{\prime}\right)\right) & =\int_{0}^{1}\left|f_{*}\left(\dot{p}_{t}\right)\right|\left(p_{t}\right) d t \\
& \leq r \int_{0}^{1}|\nabla f|\left(p_{t}\right) d t
\end{aligned}
$$

Integrate this inequality over $p^{\prime} \in B_{\frac{r}{2}}\left(\bar{p}_{\frac{1}{2}}\right)$ and we obtain

$$
\begin{aligned}
& \int_{B_{\frac{r}{2}}\left(\bar{p}_{\frac{1}{2}}\right)} d\left(f(p), f\left(p^{\prime}\right)\right) d \mu\left(p^{\prime}\right) \\
& \leq \int_{B_{\frac{r}{2}}\left(\bar{p}_{\frac{1}{2}}\right)}\left(r \int_{0}^{1}|\nabla f|\left(p_{t}\right) d t\right) d \mu\left(p^{\prime}\right) \\
& \leq r \int_{0}^{1} \frac{1}{t^{2}} \int_{B_{\frac{r t}{2}}\left(\bar{p}_{\frac{t}{2}}\right)}|\nabla f| d \mu(w) d t \\
& \leq r \int_{0}^{1} \frac{1}{t^{2}} \int_{B_{r t}(p)}|\nabla f| d \mu(w) d t \\
& \leq r \int_{0}^{1} \frac{1}{t^{2}}\left(\operatorname{Vol}\left(B_{r t}(p)\right)\right)^{1 / 2} \cdot\left(\int_{B_{r t}(p)}|\nabla f|^{2} d \mu\right)^{1 / 2} d t \\
& \leq r \int_{0}^{1} \frac{1}{t^{2}} C(r t) \cdot \frac{\sqrt{E^{f}}}{r_{0}^{\alpha}}(r t)^{\alpha} d t \\
& =\frac{C \sqrt{E^{f}} r^{2+\alpha}}{r_{0}^{\alpha}} \int_{0}^{1} t^{\alpha-1} d t \\
& =\frac{C \sqrt{E^{f}}}{\alpha r_{0}^{\alpha}} r^{2+\alpha},
\end{aligned}
$$

where $C$ is a constant dependent on $n$, by applying inequality (3.10). Similarly,

$$
\int_{B_{\frac{r}{2}}\left(\bar{p}_{\frac{1}{2}}\right)} d\left(f\left(p^{\prime}\right), f(q)\right) d \mu\left(p^{\prime}\right)=\frac{C \sqrt{E^{f}}}{\alpha r_{0}^{\alpha}} r^{2+\alpha} .
$$


By the triangle inequality,

$$
\begin{aligned}
& \int_{B_{\frac{r}{2}}\left(\bar{p}_{\frac{1}{2}}\right)} d(f(p), f(q)) d \mu\left(p^{\prime}\right) \\
& \quad \leq \int_{B_{\frac{r}{2}}\left(\bar{p}_{\frac{1}{2}}\right)} d\left(f(p), f\left(p^{\prime}\right)\right) d \mu\left(p^{\prime}\right)+\int_{B_{\frac{r}{2}}\left(\bar{p}_{\frac{1}{2}}\right)} d\left(f\left(p^{\prime}\right), f(q)\right) d \mu\left(p^{\prime}\right) \\
& \leq \frac{2 C \sqrt{E f}}{\alpha r_{0}^{\alpha}} r^{2+\alpha}
\end{aligned}
$$

and thus

$$
d(f(p), f(q)) \leq \frac{2 C \sqrt{E^{f}} r^{2}}{\alpha r_{0}^{\alpha} \operatorname{Vol}\left(B_{\frac{r}{2}}\left(\bar{p}_{\frac{1}{2}}\right)\right)} \cdot r^{\alpha}
$$

and this is the desired result.

Because of the assumption on $X$, we can prove a much stronger regularity result for harmonic maps than the local Hölder continuity derived above. First, we prove that a harmonic map is locally Lipschitz away from the vertices. Because this is a local theory, we restrict our attention to a local model $X_{1}$ defined in Section 2.2. Before we proceed with the proof of the regularity result, we show the following mean value inequality and maximum principle for a distance function between two harmonic maps.

Proposition 3.8. Let $f, g: X \rightarrow(Y, d)$ be harmonic maps into a $N P C$ space. For any $0<r_{0}<r \leq 1$, there exists $c>0$ (dependent only on $r_{0}$ and $r$ ) so that for any $p_{0} \in X$ with $r<\varrho\left(p_{0}\right)=\sup \left\{r: B_{r}\left(p_{0}\right) \subset \operatorname{st}\left(p_{0}\right)\right\}$,

$$
\max _{p \in B_{r_{0}}\left(p_{0}\right)} d^{2}(f, g)(p) \leq c \int_{B_{r}\left(p_{0}\right)} d^{2}(f, g) d \mu
$$

and

$$
\max _{p \in B_{r_{0}}\left(p_{0}\right)} d^{2}(f, g)(p) \leq c \int_{\partial B_{r}\left(p_{0}\right)} d^{2}(f, g) d \mu .
$$

Furthermore, for any $p_{0} \in X$, we have

$$
\max _{B_{r}\left(p_{0}\right)} d(f, g)=\max _{\partial B_{r}\left(p_{0}\right)} d(f, g) .
$$

Proof. If $p_{0}$ is a face point, then the existence of a sufficiently large $c$ to satisfy inequality (3.11) follows from [KS1] Lemma 2.4.2. We must also find a sufficiently large $c$ so that inequality (3.11) holds when $p_{0}$ is a edge point or when 
$p_{0}$ is a vertex. It is sufficient to consider harmonic maps $f, g: X_{r} \rightarrow(Y, d)$ (to handle the case when $p_{0}$ is an edge point) or $f, g: X_{r}^{\prime} \rightarrow(Y, d)$ (to handle the case when $p_{0}$ is a vertex), $0<r_{0}<r \leq 1$ and $p_{0}=0$. (Refer to Section 2.2 for the definitions of $X_{r}$ and $X_{r}^{\prime}$.)

For the case when the domain of $f$ and $g$ is $X_{r}=\cup_{i=1}^{n} D_{r, i}^{+} / \sim$, let $\Omega_{r}=$ $\cup_{i=1}^{n} D_{r, i} / \approx$ where the equivalence relation $\approx$ identifies the $x$-axis of one disk to that of another, i.e. $\left(x_{i}, 0\right) \approx\left(x_{j}, 0\right)$. (Here, we again use the convention that the point with coordinates $(x, y)$ on the $i$ th disk $D_{r, i}$ is denoted by $\left(x_{i}, y_{i}\right)$.) Define $\phi_{i}: D_{r, i} \rightarrow D_{r, i}^{+}$by setting $\phi_{i}\left(x_{i}, y_{i}\right)=\left(x_{i},\left|y_{i}\right|\right)$ and let $F, G: \Omega_{r} \rightarrow$ $(Y, d)$ be defined by $F\left(x_{i}, y_{i}\right)=f \circ \phi\left(x_{i}, y_{i}\right)$, and $G\left(x_{i}, y_{i}\right)=g \circ \phi\left(x_{i}, y_{i}\right)$ respectively.

For $\eta \in C_{c}^{\infty}\left(D_{r}\right)$, define $F_{\eta}, F_{1-\eta}: \Omega_{r} \rightarrow(Y, d)$ by setting

$$
F_{\eta}\left(x_{i}, y_{i}\right)=\left(1-\eta\left(x_{i}, y_{i}\right)\right) F\left(x_{i}, y_{i}\right)+\eta\left(x_{i}, y_{i}\right) G\left(x_{i}, y_{i}\right)
$$

and

$$
F_{1-\eta}\left(x_{i}, y_{i}\right)=\eta\left(x_{i}, y_{i}\right) F\left(x_{i}, y_{i}\right)+\left(1-\eta\left(x_{i}, y_{i}\right)\right) G\left(x_{i}, y_{i}\right)
$$

where $(1-t) P+t Q$ denotes the point which is fraction $t$ of the way along the geodesic from $P$ to $Q$ in $Y$. These maps are well-defined with respect to the equivalence relation $\approx$ since

$$
\begin{aligned}
F_{\eta}\left(x_{i}, 0\right) & =\left(1-\eta\left(x_{i}, 0\right)\right) F\left(x_{i}, 0\right)+\eta\left(x_{i}, 0\right) G\left(x_{i}, 0\right) \\
& =\left(1-\eta\left(x_{i}, 0\right)\right) f \circ \Phi\left(x_{i}, 0\right)+\eta\left(x_{i}, 0\right) g \circ \Phi\left(x_{i}, 0\right) \\
& =\left(1-\eta\left(x_{j}, 0\right)\right) f \circ \Phi\left(x_{j}, 0\right)+\eta\left(x_{j}, 0\right) g \circ \Phi\left(x_{j}, 0\right) \\
& =\left(1-\eta\left(x_{j}, 0\right)\right) F\left(x_{j}, 0\right)+\eta\left(x_{j}, 0\right) G\left(x_{j}, 0\right) \\
& =F_{\eta}\left(x_{j}, 0\right) .
\end{aligned}
$$

By Lemma 2.4.1 of [KS1] and by Lemma 2.4.2 of [KS1] applied to the restriction of $F_{\eta}$ and $F_{1-\eta}$ to $D_{r, i}^{+}$for each $i$, we see that $F_{\eta}, F_{1-\eta} \in W^{1,2}\left(\Omega_{r}, Y\right)$ and

$$
\begin{aligned}
& \int_{\Omega_{r}}\left|\nabla F_{\eta}\right|^{2}+\int_{\Omega_{r}}\left|\nabla F_{1-\eta}\right|^{2} \\
& \quad \leq \int_{\Omega_{r}}|\nabla F|^{2}+\int_{\Omega_{r}}|\nabla G|^{2}-2 \int_{\Omega_{r}} \nabla \eta \cdot \nabla d^{2}(F, G)+\int_{\Omega_{r}} Q(\eta, \nabla \eta),
\end{aligned}
$$

where $Q(\eta, \nabla \eta)$ consists of integrable terms which are quadratic in $\eta$ and $\nabla \eta$. Let $f_{\eta}, f_{1-\eta}$ be defined by $F_{\eta} \circ \Phi^{-1}, F_{1-\eta} \circ \Phi^{-1}$ respectively, where $\Phi^{-1}: X_{r} \rightarrow$ $\Omega_{r}$ is defined $\left(x_{i}, y_{i}\right) \mapsto\left(x_{i}, y_{i}\right)$. Since $\Phi^{-1}$ is a really just the identify map,

$$
\int_{\Omega_{r}}|\nabla F|^{2}=2 \int_{X_{r}}|\nabla f|^{2} \leq 2 \int_{X_{r}}\left|\nabla f_{\eta}\right|^{2}=\int_{\Omega_{r}}\left|\nabla F_{\eta}\right|^{2}
$$


and

$$
\int_{\Omega_{r}}|\nabla G|^{2}=2 \int_{X_{r}}|\nabla g|^{2} \leq 2 \int_{X_{r}}\left|\nabla f_{1-\eta}\right|^{2}=\int_{\Omega_{r}}\left|\nabla F_{1-\eta}\right|^{2} .
$$

Therefore,

$$
-2 \int_{\Omega_{r}} \nabla \eta \cdot \nabla d^{2}(F, G)+\int_{\Omega_{r}} Q(\eta, \nabla \eta) \geq 0
$$

and replacing $\eta$ by $t \eta$, dividing by $t$ and letting $t \rightarrow 0$, we get

$$
-\int_{D_{r}} \nabla \eta \cdot \nabla \delta \geq 0
$$

for any $\eta \in C_{c}^{\infty}\left(D_{r}\right)$ and where

$$
\delta(x, y)=\sum_{i=1}^{n} d^{2}\left(F\left(x_{i}, y_{i}\right), G\left(x_{i}, y_{i}\right)\right) .
$$

By the mean value inequality for subharmonic functions,

$$
\delta(x, y) \leq \frac{1}{\pi \rho^{2}} \int_{D_{\rho}(x, y)} \delta
$$

for any $\rho \leq r-\sqrt{x^{2}+y^{2}}$ where $D_{\rho}(x, y)$ is the disk of radius $\rho$ centered at $(x, y)$. (As before, $D_{r}$ is the disk of radius $r$ centered at the origin.)

Recall that $r_{0}<r \leq 1$. Let $\left(x_{i}, y_{i}\right) \in X_{r_{0}}$ and $\rho=r-r_{0}$.

$$
\begin{aligned}
d^{2}\left(f\left(x_{i}, y_{i}\right), g\left(x_{i}, y_{i}\right)\right) & =d^{2}\left(F\left(x_{i}, y_{i}\right), G\left(x_{i}, y_{i}\right)\right) \\
& \leq \delta(x, y) \\
& \leq \frac{1}{\pi \rho^{2}} \int_{D_{\rho}(x, y)} \delta \\
& \leq \frac{1}{\pi \rho^{2}} \int_{D_{r}} \delta \\
& =\frac{1}{\pi \rho^{2}} \int_{\Omega_{r}} d^{2}(F, G) \\
& =\frac{2}{\pi \rho^{2}} \int_{X_{r}} d^{2}(f, g) .
\end{aligned}
$$

This proves inequality (3.11).

For the case when the domain of $f$ and $g$ is $X_{r}^{\prime}=\cup_{i=1}^{n} W_{i} / \sim$, let $\psi_{i}$ : $D_{r^{3}, i}^{+} \rightarrow W_{i}$ be defined by $z \mapsto z^{\frac{1}{3}}$. Let $\Omega_{r^{3}}=\cup_{i=1}^{n} D_{r^{3}, i} / \approx$ where $\approx$ is defined as before. Let $\phi_{i}: D_{r^{3}, i} \rightarrow D_{r^{3}, i}^{+}$be the map $\phi_{i}\left(x_{i}, y_{i}\right)=\left(x_{i},\left|y_{i}\right|\right)$ and define 
$\Psi: \Omega_{r^{3}} \rightarrow X_{r}^{\prime}$ by setting $\Psi\left(x_{i}, y_{i}\right)=\psi_{i} \circ \phi_{i}\left(x_{i}, y_{i}\right)$ and let $F, G: \Omega_{r^{3}} \rightarrow(Y, d)$ be defined by $f \circ \Psi$, and $g \circ \Psi$ respectively. Arguing analogously as above, we also get inequality (3.14). Here, we note that

$$
\int_{\Omega_{r^{3}}}|\nabla F|^{2}=2 \int_{X_{r}^{\prime}}|\nabla f|^{2} \leq 2 \int_{X_{r}^{\prime}}\left|\nabla f_{\eta}\right|^{2}=\int_{\Omega_{r^{3}}}\left|\nabla F_{\eta}\right|^{2}
$$

and

$$
\int_{\Omega_{r^{3}}}|\nabla G|^{2}=2 \int_{X_{r}^{\prime}}|\nabla g|^{2} \leq 2 \int_{X_{r}}\left|\nabla f_{1-\eta}\right|^{2}=\int_{\Omega_{r^{3}}}\left|\nabla F_{1-\eta}\right|^{2}
$$

follows from the conformal invariance of energy.

Again suppose $r_{0}<\theta_{0} \leq r \leq 1$. Let $\left(x_{i}, y_{i}\right) \in X_{r_{0}}$ and $\rho=\theta_{0}^{3}-r_{0}^{3}$. Using the notation $z=x+i y$ and $z^{3}=\xi+i \eta$,

$$
\begin{aligned}
d^{2}\left(f\left(x_{i}, y_{i}\right), g\left(x_{i}, y_{i}\right)\right) & =d^{2}\left(F\left(\xi_{i}, \eta_{i}\right), G\left(\xi_{i}, \eta_{i}\right)\right) \\
& \leq \delta(\xi, \eta) \\
& \leq \frac{1}{\pi \rho^{2}} \int_{D_{\rho}(\xi, \eta)} \delta \\
& =\frac{1}{\pi \rho^{2}} \int_{D_{r^{3}}} \delta \\
& =\frac{1}{\pi \rho^{2}} \int_{\Omega_{r^{3}}} d^{2}(F, G) . \\
& =\frac{2}{\pi \rho^{2}} \int_{\left\{\left(x_{i}, y_{i}\right) \in \Omega_{r^{3}}: y_{i} \geq 0\right\}} d^{2}(F, G) .
\end{aligned}
$$

By a change of variables $R=r^{3}$ and $\Theta=3 \theta$ (i.e. $z=r e^{i \theta} \mapsto z^{3}=R e^{i \Theta}$ ),

$$
\begin{aligned}
\int_{D_{r^{3}, i}^{+}} d^{2}(F, G) & =\int_{0}^{\pi} \int_{0}^{r^{3}} d^{2}(F, G) R d R d \Theta \\
& =9 \int_{0}^{\frac{\pi}{3}} \int_{0}^{r} d^{2}(f, g) r^{5} d r d \theta \\
& \leq 9 \int_{0}^{\frac{\pi}{3}} \int_{0}^{r} d^{2}(f, g) r d r d \theta \\
& =9 \int_{W_{i}} d^{2}(f, g)
\end{aligned}
$$

and hence

$$
d^{2}\left(f\left(x_{i}, y_{i}\right), g\left(x_{i}, y_{i}\right)\right) \leq \frac{18}{\pi \rho^{2}} \int_{X_{r}^{\prime}} d^{2}(f, g)
$$


for any $\left(x_{i}, y_{i}\right) \in X_{r_{0}}$. This proves inequality (3.11). Inequality (3.12) follows from a similar argument.

To prove the maximum principle (equality (3.13)), suppose the maximum of $d(f, g)$ in $X_{r}$ or $X_{r}^{\prime}$ is achieved at 0 . Since $\delta(0)=n d(f(0), g(0))$, then the maximum of $\delta$ in $D_{r}$ is also achieved at 0 . Inequality 3.14, implies that $\delta$ is identically equal to $\delta(0)$ and this is impossible unless $d(f, g)$ is identically constant on $X_{r}$.

To prove the Lipschitz regularity, we will use the following property of harmonic maps along the edges. The example of when the domain was a disk was examined in the introduction.

Theorem 3.9. Let $f: X_{1} \rightarrow Y$ be a harmonic map where $X_{1}$ is an edge piece (defined in Section 2.2) with $N$ half disks labelled $D_{1}^{+}, \ldots, D_{N}^{+}$and $Y$ is an NPC space. Let $f^{j}=\left.f\right|_{D_{j}^{+}}$and fix a conformal structure on each $D_{j}^{+}$so that the orientation induced on the $x$-axis is the same for each $j=1, \ldots, N$. With this conformal structure, define

$$
\varphi_{j}=\left|\frac{\partial f^{j}}{\partial x}\right|^{2}-\left|\frac{\partial f^{j}}{\partial y}\right|^{2}-2 i\left\langle\frac{\partial f^{j}}{\partial x} \cdot \frac{\partial f^{j}}{\partial y}\right\rangle .
$$

Then

$$
\operatorname{Im} \sum_{j=1}^{N} \varphi_{j}(x, 0)=0
$$

for all $-1<x<1$.

Proof. Let $\Phi_{t}: D^{+} \rightarrow D^{+}$be a diffeomorphism so that $\Phi_{0}=$ identity and $\Phi_{t}=$ identity near $\partial D \cap D^{+}$. Set $\frac{\partial \Phi_{t}}{\partial t}=\left(\nu_{t}, \omega_{t}\right)$ and $\nu=\nu_{0}$ and $\omega=\omega_{0}$. For $\Phi_{t}$ with compact support in $D^{+}$, the standard variational computation for $E\left(f^{j} \circ \Phi_{t}\right)$ using the fact that $f^{j}$ is minimizing in $D^{+}$gives

$$
\begin{aligned}
0= & \left.\frac{d}{d t} E\left(f^{i} \circ \Phi_{t}\right)\right|_{t=0} \\
= & \int_{D}\left(\left|\frac{\partial f^{j}}{\partial x}\right|^{2}-\left|\frac{\partial f^{j}}{\partial y}\right|^{2}\right)\left(\frac{\partial \nu}{\partial x}-\frac{\partial \omega}{\partial y}\right) \\
& +2\left\langle\frac{\partial f^{j}}{\partial x} \cdot \frac{\partial f^{j}}{\partial y}\right\rangle\left(\frac{\partial \nu}{\partial y}-\frac{\partial \omega}{\partial x}\right) d x d y
\end{aligned}
$$

and we conclude $\varphi_{j}$ is holomorphic in the interior of $D^{+}$. 
Now consider $\Phi_{t}$ with $\omega_{t} \equiv 0$ near $\Gamma=\{(x, y) \mid y=0,-1<x<1\}$. Let $\bar{\Phi}_{t}: X_{1} \rightarrow X_{1}$ be defined by $\bar{\Phi}_{t}\left(x_{i}, y_{i}\right)=\Phi_{t}(x, y)$. Using the fact that $f$ is energy minimizing and that $f \circ \bar{\Phi}_{t}$ is a competitor of $f$, we obtain

$$
\begin{aligned}
0= & \left.\frac{d}{d t} E\left(f \circ \bar{\Phi}_{t}\right)\right|_{t=0} \\
= & \sum_{j=1}^{n} \int_{D}\left(\left|\frac{\partial f^{j}}{\partial x}\right|^{2}-\left|\frac{\partial f^{j}}{\partial y}\right|^{2}\right)\left(\frac{\partial \nu}{\partial x}-\frac{\partial \omega}{\partial y}\right) \\
& +2\left\langle\frac{\partial f^{j}}{\partial x} \cdot \frac{\partial f^{j}}{\partial y}\right\rangle\left(\frac{\partial \nu}{\partial y}-\frac{\partial \omega}{\partial x}\right) d x d y
\end{aligned}
$$

We now assume that $\omega_{t} \equiv 0$ and $\nu_{t}$ a smooth function with support in $\left(-x_{0}, x_{0}\right) \times\left[0, y_{0}\right) \subset D^{+}$. Let

$$
U=\sum_{j=1}^{n}\left(\left|\frac{\partial f^{j}}{\partial x}\right|^{2}-\left|\frac{\partial f^{j}}{\partial y}\right|^{2}\right) \text { and } V=2 \sum_{i=1}^{n}\left\langle\frac{\partial f^{j}}{\partial x} \cdot \frac{\partial f^{j}}{\partial y}\right\rangle .
$$

Then, from the fact that $\omega_{t} \equiv 0$ and by integration by parts,

$$
\begin{aligned}
0= & \lim _{\delta \rightarrow 0} \int_{\delta}^{y_{0}} \int_{-x_{0}}^{x_{0}} U \frac{\partial \nu}{\partial x} d x d y-\int_{-x_{0}}^{x_{0}} \int_{\delta}^{y_{0}} V \frac{\partial \nu}{\partial y} d y d x \\
= & \lim _{\delta \rightarrow 0} \int_{\delta}^{y_{0}}\left(U \nu\left(x_{0}, y\right)-U \nu\left(-x_{0}, y\right)-\int_{-x_{0}}^{x_{0}} \frac{\partial U}{\partial x} \nu d x\right) d y \\
& -\lim _{\delta \rightarrow 0} \int_{-x_{0}}^{x_{0}}\left(V \nu\left(x, y_{0}\right)-V \nu(x, \delta)-\int_{\delta}^{y_{0}} \frac{\partial V}{\partial y} \nu d y\right) d x
\end{aligned}
$$

Using that spt $\nu \subset\left(-x_{0}, x_{0}\right) \times\left[0, y_{0}\right)$ and the Cauchy-Riemann equation $\left(\frac{\partial U}{\partial x}=\frac{\partial V}{\partial y}\right)$, we obtain

$$
0=\lim _{\delta \rightarrow 0} \int_{-x_{0}}^{x_{0}} V \nu(x, \delta) d x
$$

Let $\psi$ be a holomorphic function in $D^{+}$so that $\frac{d \psi}{d z}=\varphi=U+i V$. Then $V=$ $\operatorname{Im} \varphi=\operatorname{Im} \frac{d \psi}{d z}=\operatorname{Im} \frac{d \psi}{d x}$. Hence

$$
0=\lim _{\delta \rightarrow 0} \operatorname{Im} \int_{-x_{0}}^{x_{0}} \frac{\partial \psi}{\partial x} \nu(x, \delta) d x=\lim _{\delta \rightarrow 0} \operatorname{Im} \int_{-x_{0}}^{x_{0}} \psi \frac{\partial \nu}{\partial x}(x, \delta) d x .
$$

Since $\frac{d \psi}{d z}=\varphi \in L^{1}\left(D^{+}\right), \psi \in H^{1,1}\left(D^{+}\right)$and $\psi$ has boundary values in $L^{1}$. Thus,

$$
0=\operatorname{Im} \int_{-x_{0}}^{x_{0}} \psi \frac{\partial \nu}{\partial x}(x, 0) d x
$$


Since $\nu$ is an arbitrary function of $\Gamma$, we conclude $\operatorname{Im} \psi=0$ on $\Gamma$. By the reflection principle, $\psi$ can be extended to a holomorphic function in the interior of $D$, and in Thus, particular, $\psi$ is smooth across $\Gamma$.

$$
0=\operatorname{Im} \int_{-x_{0}}^{x_{0}} \frac{d \psi}{d z} \nu(x, 0) d x=\operatorname{Im} \int_{-x_{0}}^{x_{0}} \varphi \nu(x, 0) d x
$$

With this, we are ready to prove the main regularity theorem.

Theorem 3.10. If $f: X_{1} \rightarrow Y$ is a harmonic map into a NPC space, then

$$
\left|\frac{\partial f}{\partial x}\right|^{2} \leq \frac{2}{\pi r^{2}} E^{f} \text { and }\left|\frac{\partial f}{\partial y}\right|^{2} \leq \frac{2 N+2}{\pi r^{2}} E^{f}
$$

at $\left(\bar{x}_{i}, \bar{y}_{i}\right) \in X_{1}$ where $2 r$ is the distance of $\left(\bar{x}_{i}, \bar{y}_{i}\right)$ to $\partial X_{1}$ and $N$ is the number of faces of $X_{1}$. (Here, $X_{1}$ is the edge piece of diameter 1 as defined in Section 2.2.)

Proof. We first prove an upper bound for $\left|\frac{\partial f}{\partial x}\right|^{2}$. We will use the notation $f^{i}(x, y)=f\left(x_{i}, y_{i}\right)$. For $\epsilon>0$ sufficiently small, let $g: X_{r} \rightarrow Y$ be defined by $g\left(x_{i}, y_{i}\right)=f^{i}(x+\epsilon, y)$. By inequality (3.14) applied to $f$ restricted to $X_{r}$ and $g$, we see that

$\sum_{j=1}^{n} d^{2}\left(f^{j}(\bar{x}, \bar{y}), f^{j}(\bar{x}+\epsilon, \bar{y})\right) \leq \frac{1}{\pi r^{2}} \int_{D_{r}(\bar{x}, \bar{y})} \sum_{j=1}^{n} d^{2}\left(f^{j}(x, y), f^{j}(x+\epsilon, y)\right) d x d y$.

Thus,

$$
\begin{aligned}
\left|\frac{\partial f}{\partial x}\right|^{2}\left(\bar{x}_{i}, \bar{y}_{i}\right) & =\lim _{\epsilon \rightarrow 0} \frac{d^{2}\left(f^{i}(\bar{x}, \bar{y}), f^{i}(\bar{x}+\epsilon, \bar{y})\right.}{\epsilon^{2}} \\
& \leq \lim _{\epsilon \rightarrow 0} \sum_{j=1}^{n} \frac{d^{2}\left(f^{j}(\bar{x}, \bar{y}), f^{j}(\bar{x}+\epsilon, \bar{y})\right.}{\epsilon^{2}} \\
& \leq \frac{1}{\pi r^{2}} \sum_{j=1}^{n} \lim _{\epsilon \rightarrow 0} \frac{1}{\epsilon^{2}} \int_{D_{r}(\bar{x}, \bar{y})} d^{2}\left(f^{j}(x, y), f^{j}(x+\epsilon, y)\right) d x d y \\
& \leq \frac{1}{\pi r^{2}} \sum_{j=1}^{n} \int_{D_{r}(\bar{x}, \bar{y})}\left|\nabla f^{j}\right|^{2} d x d y \\
& \leq \frac{2}{\pi r^{2}} \int_{X_{1}}|\nabla f|^{2} d \mu .
\end{aligned}
$$


Next, we prove an upper bound for $\left|\frac{\partial f}{\partial y}\right|^{2}$. Let $\varphi=\sum_{j=1}^{N} \varphi_{j}$ where $\varphi_{j}$ is as in Theorem 3.9. By Theorem $3.9 \operatorname{Im} \varphi=0$ on $\Gamma$ and $\varphi$ can be extended to a holomorphic function in the interior of $D$. Because $\operatorname{Re} \varphi$ is harmonic, the mean value inequality gives

$$
\operatorname{Re} \varphi(\bar{x}, \bar{y})=\frac{1}{\pi r^{2}} \int_{D_{r}(\bar{x}, \bar{y})} \operatorname{Re} \varphi d x d y
$$

for $(\bar{x}, \bar{y}) \in D$ and $2 r=1-\sqrt{\bar{x}^{2}+\bar{y}^{2}}$. Thus,

$$
\begin{aligned}
|\operatorname{Re} \varphi|(\bar{x}, \bar{y}) & \leq \frac{1}{\pi r^{2}} \int_{D_{r}(\bar{x}, \bar{y})}|\operatorname{Re} \varphi| d x d y \\
& \leq \frac{1}{\pi r^{2}} \int_{D}|\operatorname{Re} \varphi| d x d y \\
& =\frac{2}{\pi r^{2}} \int_{D^{+}}|\operatorname{Re} \varphi| d x d y .
\end{aligned}
$$

Using the fact that

$$
|\operatorname{Re} \varphi|=|U| \leq \sum_{i=1}^{n}\left|\nabla f^{j}\right|^{2}
$$

we have

$$
|\operatorname{Re} \varphi|(\bar{x}, \bar{y}) \leq \frac{2}{\pi r^{2}} \sum_{i=1}^{n} \int_{D^{+}}\left|\nabla f^{i}\right|^{2}=\frac{2}{\pi r^{2}} \int_{X_{1}}|\nabla f|^{2}
$$

Thus,

$$
\begin{aligned}
\left|\frac{\partial f}{\partial y}\right|^{2}\left(\bar{x}_{i}, \bar{y}_{i}\right) & =\left|\frac{\partial f^{i}}{\partial y}\right|^{2}(\bar{x}, \bar{y}) \\
& \leq \sum_{j=1}^{n}\left|\frac{\partial f^{j}}{\partial y}\right|^{2}(\bar{x}, \bar{y}) \\
& =\sum_{j=1}^{n}\left|\frac{\partial f^{j}}{\partial x}\right|^{2}(\bar{x}, \bar{y})-\operatorname{Re} \varphi(\bar{x}, \bar{y}) \\
& \leq \sum_{j=1}^{n}\left|\frac{\partial f^{j}}{\partial x}\right|^{2}(\bar{x}, \bar{y})+|\operatorname{Re} \varphi|(\bar{x}, \bar{y}) \\
& \leq \frac{2 n+2}{\pi r^{2}} \int_{X_{1}}|\nabla f|^{2} .
\end{aligned}
$$

As a consequence of Theorem 3.10, we have the following. 
Theorem 3.11. Let $X$ and $Y$ as above and $V$ be the set of vertices of $X$. If $f: X \rightarrow Y$ is a harmonic map, then for every $p \in X-V$, there is a constant $c$ independent of $p$ so that

$$
|\nabla f|^{2}(p) \leq \frac{c}{\delta^{2}} \int_{B_{\delta}(p)}|\nabla f|^{2} d \mu
$$

where $\delta=\min _{v \in V} d_{X}(p, v)$ and $f$ is locally Lipschitz continuous with the Lipschitz constant at $p \in X$ dependent only on $E^{f}$ and $\delta$.

Proof. For $p \in X-V$, let $v$ be a vertex so that $\delta=d_{X}(p, v)$. Furthermore, let $q$ be an edge point closest to $p$ and let $\rho=d_{X}(q, v)$. If $\theta$ is the angle between the edge containing $q$ and the line segment from $p$ to $v$, then $\rho=\delta \cos \theta \geq \frac{\delta}{\sqrt{2}}$ since $\theta \leq \frac{\pi}{6}$ by the assumption that the faces of $X$ are isometric to an equilateral triangle. Furthermore, the distance from $q$ to an edge not containing $q$ is equal to $\rho \sin \frac{\pi}{3} \geq \frac{\delta}{2}$. Therefore, $B_{\frac{\delta}{2}}(q)$ is isometric to the edge piece $X_{1}$ modulo a dilation. Now restricting $f$ to $B_{\frac{\delta}{2}}(q)$, noting that the energy of $f$ is independent of a conformal change of variables on each face and applying Theorem 3.10, we have

$$
\begin{aligned}
|\nabla f|^{2}(p) & =\left|\frac{\partial f}{\partial x}\right|^{2}(p)+\left|\frac{\partial f}{\partial x}\right|^{2}(p) \\
& \leq \frac{4}{\pi\left(\frac{\delta}{2}\right)^{2}} \int_{B_{\frac{\delta}{2}}(q)}|\nabla f|^{2} d \mu \\
& \leq \frac{c}{\delta^{2}} \int_{B_{\delta}(p)}|\nabla f|^{2} d \mu .
\end{aligned}
$$

The Lipschitz regularity of $f$ follows immediately.

We now improve the local Hölder continuity result of Theorem 3.7 to prove the uniform Hölder continuity of harmonic maps. [F] has claimed that harmonic map $f: X \rightarrow Y$ is locally uniformly Hölder continuous; that is, there exists constants $A, \alpha, \delta$ so that

$$
d(f(p), f(q)) \leq A d_{X}^{\alpha}(p, q) \text { whenever } d_{X}(p, q)<\delta
$$

with $A, \alpha, \delta$ independent of $p$ locally. We note that $[\mathrm{F}]$ considers more general metrics that we are considering in this paper. On the other hand, we prove a slightly stronger statement. 
Theorem 3.12. Let $X$ and $Y$ as above. If $f: X \rightarrow Y$ is a harmonic map, then $f$ is globally Hölder continuous. More specifically, there exist constants $A$ and $\alpha$ depending only on $E^{f}$ and $X$ so that

$$
d(f(p), f(q)) \leq A d_{X}^{\alpha}(p, q) \text { whenever } d_{X}(p, q) \leq 1
$$

Proof. Note that by definition of $X, \varrho(v)=\sqrt{3}$ for all $v \in V$. By Theorem 3.7, we know that for each $v \in V$, there exists $A_{v}$ and $\alpha_{v}$ depending on $E^{f}$ and $v$ so that

$$
d(f(p), f(v)) \leq A_{v} d_{X}^{\alpha_{v}}(p, v)
$$

for all $p \in B_{\sqrt{3}}(v)$. Let

$$
A_{0}=\max _{v \in V} A_{v} \text { and } \alpha_{0}=\min _{v \in V} \alpha_{v}
$$

Let $p, q \in X$ with $d_{X}(p, q) \leq 1, t \mapsto p_{t}$ for $t \in[0,1]$, be a constant speed geodesic from $p$ to $q$ in $X$ and $r=d(p, q)$.

CASE I: $\left\{p_{t}: t \in[0,1]\right\} \cap B_{r}(v)=\emptyset$ for all $v \in V$.

Fix $t \in[0,1]$, let $v_{0} \in V$ be so that $d_{X}\left(p_{t}, v_{0}\right)=\min _{v \in V} d_{X}\left(p_{t}, V\right)$ and $\sigma=d\left(p_{t}, v_{0}\right)$. By Theorem 3.11,

$$
\begin{aligned}
|\nabla f|^{2}\left(p_{t}\right) & \leq \frac{c}{\sigma^{2}} \int_{B_{\sigma}\left(p_{t}\right)}|\nabla f|^{2} \\
& \leq \frac{c}{\sigma^{2}} \int_{B_{2 \sigma}\left(v_{0}\right)}|\nabla f|^{2} \\
& =\frac{4 c}{(2 \sigma)^{2}} \int_{B_{2 \sigma}\left(v_{0}\right)}|\nabla f|^{2} .
\end{aligned}
$$

If $\sigma>\sqrt{r}$ then by inequality (3.16),

$$
|\nabla f|^{2}\left(p_{t}\right) \leq \frac{c}{r} \int_{B_{\sigma}\left(p_{t}\right)}|\nabla f|^{2} \leq \frac{c}{r} E^{f} .
$$

Now assume $\sigma \leq \sqrt{r}$ and let $E(r)=\int_{B_{r}\left(v_{0}\right)}|\nabla f|^{2} d \mu$ and $I(r)=$ $\int_{\partial B_{r}\left(v_{0}\right)} d^{2}(f, f(v)) d s$. We consider the following two possibilities:

(i) $\frac{2 \sigma E(2 \sigma)}{I(2 \sigma)} \geq 1$ 
Then by Corollary 3.5,

$$
\frac{E(2 \sigma)}{(2 \sigma)^{2}} \leq \frac{E(1)}{1} \leq E^{f}
$$

and thus by inequality (3.18)

$$
|\nabla f|^{2}\left(p_{t}\right) \leq 4 c E^{f}
$$

(ii) $\frac{2 \sigma E(2 \sigma)}{I(2 \sigma)}<1$

Since $d^{2}\left(f(p), f\left(v_{0}\right)\right) \leq A_{0}^{2}(2 \sigma)^{2 \alpha_{0}}$ for $p \in \partial B_{2 \sigma}\left(v_{0}\right)$,

$$
E(2 \sigma) \leq \frac{I(2 \sigma)}{2 \sigma} \leq \frac{A_{0}^{2}(2 \sigma)^{2 \alpha_{0}+1}}{2 \sigma} \leq A_{0}^{2} 4^{\alpha_{0}} \sigma^{2 \alpha_{0}} .
$$

Thus, inequality (3.17) and the fact that $r \leq \sigma \leq \sqrt{r}$,

$$
\begin{aligned}
|\nabla f|^{2}\left(p_{t}\right) & \leq c A_{0}^{2} 4^{\alpha_{0}} \sigma^{2 \alpha_{0}-2} \\
& \leq c A_{0}^{2} 4^{\alpha_{0}} r^{\alpha_{0}-1}
\end{aligned}
$$

From inequalities (3.19), (3.20) and (3.21),

$$
|\nabla f|^{2}\left(p_{t}\right) \leq C_{1} r^{-1}+C_{2}+C_{3} r^{\alpha_{0}-1}
$$

where $C_{1}, C_{2}, C_{3}$ depends only on $E^{f}, A_{0}$ and $\alpha_{0}$. Therefore,

$$
\begin{aligned}
d^{2}(f(p), f(q)) & \leq r^{2} \int_{0}^{1}|\nabla f|^{2}\left(p_{t}\right) d t \\
& \leq C_{1} r+C_{2} r^{2}+C_{3} r^{\alpha_{0}+1}
\end{aligned}
$$

CASE II: $p_{t} \in B_{r}(v)$ for some $t \in[0,1]$ and $v \in V$.

By repeated use of the triangle inequality, we have

$$
\begin{aligned}
d(f(p), f(q)) & \leq d(f(p), f(v))+d(f(v), f(q)) \\
& \leq A_{0} d_{X}^{\alpha_{0}}(p, v)+A_{0} d_{X}^{\alpha_{0}}(v, q) \\
& \leq A_{0}\left(d_{X}\left(p, p_{t}\right)+d_{X}\left(p_{t}, v\right)\right)^{\alpha_{0}}+A_{0}\left(d_{X}\left(v, p_{t}\right)+d_{X}\left(p_{t}, q\right)\right)^{\alpha_{0}} \\
& \leq A_{0}(2 r)^{\alpha_{0}}+A_{0}(2 r)^{\alpha_{0}} \\
& \leq 2^{1+\alpha_{0}} A_{0} r^{\alpha_{0}}
\end{aligned}
$$

Choosing $A=C_{1}+C_{2}+C_{3}+2^{1+\alpha_{0}} A_{0}$ and $\alpha=\min \left\{\frac{1}{2}, \alpha_{0}\right\}$, we have the desired result. 


\section{Existence Results.}

We first start with the existence of the Dirichlet solution.

Proposition 4.1. Fix $B_{1}=D_{1}$ or $B_{1}=X_{1}$ or $B_{1}=X_{1}^{\prime}$ (cf. Section 2.2) and let $(Y, d)$ be a NPC space. Given $\phi: W^{1,2}\left(B_{1}, Y\right)$, define

$$
W_{\phi}^{1,2}=\left\{u \in W^{1,2}(B, Y) \mid \operatorname{tr}(u)=\operatorname{tr}(\phi)\right\} .
$$

Then there exists a unique harmonic map $f \in W_{\phi}^{1,2}$ and

$$
E^{f}=\inf _{u \in W_{\phi}^{1,2}} E^{u}
$$

Here $\operatorname{tr}(u) \in L^{2}(\partial B, Y)$ is the trace of the map $u$ as defined in [KS1].

Proof. Follows exactly as in [KS1] Theorem 2.2.

We prove the following boundary regularity for the Dirichlet solution.

Lemma 4.2. Let $B_{\tau}=\left\{z=\left(x_{i}, y_{i}\right) \in B_{1}:|z|=\sqrt{x_{i}^{2}+y_{i}^{2}} \leq \tau\right\}$ and $(Y, d)$ be a NPC space. Given $\phi: B_{\tau} \rightarrow Y$ which is uniformly continuous up to the boundary, the Dirichlet solution $u: B_{\tau} \rightarrow Y$ with $\left.u\right|_{\partial B_{\tau}}=\left.\phi\right|_{\partial B_{\tau}}$ is also uniformly continuous up to the boundary.

Proof. For $z \in B_{\tau}$, a straight forward modification of the proof of the CourantLebesgue lemma implies that for all $\sigma<1$, there exist $r \in(\sigma, \sqrt{\sigma})$ and a constant $C>0$ so that

$$
d\left(u\left(z_{1}\right), u\left(z_{2}\right)\right) \leq C \log \left(\frac{1}{\sigma}\right)^{-1 / 2}
$$

for all $z_{1}, z_{2} \in \partial B_{r}(z) \cap B_{\tau}$. This combined with the uniform continuity of $\phi$ implies that for all $\epsilon>0$, there exists $\delta>0$ sufficiently small so that

$$
u\left(\left(\partial B_{\delta}(z) \cap B_{\tau}\right) \cup\left(\partial B_{\tau} \cap B_{\delta}(z)\right)\right) \subset B_{\epsilon}(u(z)) .
$$

Since the image of a harmonic map is always contained in a convex hull of its boundary, this implies

$$
u\left(B_{\delta}(z) \cap B_{\tau}\right) \subset B_{\epsilon}(u(z))
$$

and this shows the uniform continuity of $u$. 
Let $\Gamma$ be a finitely generated group. Then there exists a finite 2-complex $X$ and a normal covering $p: \bar{X} \rightarrow X$ with transformation group $\Gamma$. If $\Gamma$ is finitely presented, then we may take $X$ so that $\pi_{1}(X)=\Gamma$ and $p: \tilde{X} \rightarrow X$ the universal cover, but in general, $\bar{X}$ may fail to be simply connected. By the construction in Lemma 2.1, we may assume that $X$ is an admissible finite 2complex without boundary. Moreover, we assign metrics on faces of $X$ so that $X$ is a flat admissible finite 2-complex without boundary as in Section 3.

Let $\gamma_{1}, \ldots, \gamma_{N}$ be the generators of $\Gamma$. An isometric action on $Y$ by $\Gamma$ is a homomorphism $\rho: \Gamma \rightarrow \operatorname{Isom}(Y)$ (sometimes also called a representation of $\Gamma)$. A map $f: \bar{X} \rightarrow Y$ is called $\rho$-equivariant if

$$
f(\gamma p)=\rho(\gamma) f(p) \text { for all } p \in \bar{X} \text { and for all } \gamma \in \Gamma .
$$

Here, and subsequently, we write $\rho(\gamma) f(p)$ for $\rho(\gamma)(f(p))$. The energy of a $\rho$ equivariant map $f: \bar{X} \rightarrow Y$ is given by

$$
E^{f}:=\int_{X}|\nabla f|^{2} d \mu
$$

where $X$ is identified with a fundamental domain of $\bar{X}$. A map $f: \bar{X} \rightarrow Y$ is said to be in the space $L_{\rho}^{2}(\bar{X}, Y)$ if $f$ is a $\rho$-equivariant map satisfying

$$
\int_{X} d^{2}(f, Q) d \mu<\infty
$$

for any $Q \in Y$. Define a distance function $d_{2}$ on $L_{\rho}^{2}(\bar{X}, Y)$ by

$$
d_{2}(f, g)=\int_{X} d^{2}(f(p), g(p)) d \mu(p)
$$

It is known (see [KS2] Lemma 2.1.2) that the curve $t \mapsto u_{t} \in L_{\rho}^{2}(\bar{X}, Y)$ for $t \in[0,1]$, where $u_{t}(p)=(1-t) u_{0}(p)+t u_{1}(p)$, is a geodesic and $\left(L_{\rho}^{2}(\bar{X}, Y), d_{2}\right)$ is a NPC space. We will refer to $\left\{u_{t}\right\}_{0 \leq t \leq 1}$ as the geodesic homotopy of $u_{0}$ and $u_{1}$. The set $W_{\rho}^{1,2}(\bar{X}, Y)$ is defined to be the subset $L_{\rho}^{2}(\bar{X}, Y)$ with finite energy.

Proposition 4.3. Let $X, \Gamma, \rho$ and $Y$ as above. There exists a $\rho$-equivariant globally Lipschitz map. Therefore, $W_{\rho}^{1,2}(\bar{X}, Y)$ is non-empty.

Proof. The proof of Proposition 2.6.1 of [KS1] can be followed almost verbatim to prove Proposition 4.3. 
Proposition 4.4. Let $X, \Gamma, \rho$ and $Y$ as above. There exists a sequence $\left\{u_{i}\right\}_{i=1,2, \ldots} \subset W_{\rho}^{1,2}(\bar{X}, Y)$ and constants $A, \alpha$ and $\delta$ so that $E^{u_{i}} \rightarrow E_{0}:=$ $\inf \left\{E^{f}: f \in W_{\rho}^{1,2}(\bar{X}, Y)\right\}$ and

$$
d\left(u_{i}(p), u_{i}(q)\right) \leq A d_{X}^{\alpha}(p, q) \text { whenever } d_{X}^{\alpha}(p, q)<\delta
$$

for all $i$.

Proof. We follow the argument of Theorem 2.6.4 of [KS1] making the necessary modification to account for the fact that $X$ is not a Riemannian manifold. By Proposition 4.3 , we know that $W_{\rho}^{1,2}(\bar{X}, Y)$ is nonempty and $E_{0}$ is finite. Let $\left\{v_{i}\right\} \subset W_{\rho}^{1,2}(\bar{X}, Y)$ be a minimizing sequence, i.e. $E^{v_{i}} \rightarrow E_{0}$.

Choose a finite cover of $X$ by balls $\left\{B^{1}, \ldots, B^{N}\right\}$ of radius $r<1$ and let $2 B^{j}$ denote ball of radius $2 r$ with the same center as $B^{j}$. Choose $r$ small enough so that for any $p \in X, \cup_{j \mid p \in 2 B^{j}} 2 B^{j}$ is simply connected. Let $\left\{\eta^{j}\right\}$ be a partition of unity subordinate to $B^{j}$. Lift the functions $\eta^{j}$ and the sets $B^{j}$ to functions $\bar{\eta}^{j}$ and sets $\bar{B}^{j}$ on $\bar{X}$ invariant under $\Gamma$. In each $2 \bar{B}^{j}$, let $u_{i}^{j}$ be the Dirichlet solution with boundary values equal to that of $v_{i}$. Extend $u_{i}^{j}$ outside of $2 \bar{B}^{j}$ by defining $u_{i}^{j}$ equal to $v_{i}$ there. Thus, $E^{u_{i}^{j}} \leq E^{v_{i}}$. Furthermore, by Theorem 3.12, there exist $A$ and $\alpha$ independent of $i$ so that

$$
d\left(u_{i}^{j}(p), u_{i}^{j}(q)\right) \leq A d_{X}^{\alpha}(p, q) \text { whenever } p \in \operatorname{spt}\left(\bar{\eta}^{j}\right) \subset \bar{B}^{j} \text { and } d(p, q) \leq \frac{r}{2}
$$

Consider a measure $\nu$ on the set of natural numbers $\mathbf{N}$ defined by $\nu(j)=\bar{\eta}^{j}(p)$. Define a function $\phi:(\mathbf{N}, \nu) \rightarrow Y$ by setting $\phi(j)=u_{i}^{j}(p)$. Let $u_{i}(p)$ be the center of mass of $\phi$, i.e.

$$
\int_{\mathbf{N}} d\left(\phi(j), u_{i}(p)\right) d \nu(j)=\inf _{Q \in Y} \int_{\mathbf{N}} d(\phi(j), Q) d \nu(j)
$$

By following the proof of Theorem 2.6.4 of [KS1], we can see that for sufficiently large $i$,

$$
d^{2}\left(u_{i}(p), u_{i}(q)\right) \leq 2 \sum_{j=1}^{n} \bar{\eta}^{j}(p) d^{2}\left(u_{i}^{j}(p), u_{i}^{j}(q)\right) .
$$

Thus, the regularity of $u_{j}^{i}$ follows from inequality (4.1). To show that $\left\{u_{i}\right\}$ is a minimizing sequence, we refer again to $[\mathrm{KS} 1]$.

We are now ready to prove the existence theorem of $\rho$-equivariant harmonic maps. We follow the proof of [KS2] taking into account that $X$ is not a Riemannian manifold. 
Theorem 4.5. Let $X, \Gamma, \rho$ and $Y$ as above with $(Y, d)$ a metric space of curvature bounded from above by $\kappa<0$. If $\rho$ does not fix an equivalent class of rays, then there exists a $\rho$-equivariant harmonic map.

Proof. Let $\mathcal{C}_{\epsilon} \subset L_{\rho}^{2}(\bar{X}, Y)$ be the set of uniformly Hölder continuous maps whose energy is bounded by $E_{0}+\epsilon$, where $E_{0}$ denotes the infimum of energies of maps in $L_{\rho}^{2}(\bar{X}, Y)$, and satisfies

$$
d(f(q), f(q)) \leq A d_{X}^{\alpha}(p, q) \text { whenever } d_{X}(p, q) \leq \delta
$$

for some constants $A, \alpha$, and $\delta$. By Proposition $4.4, \mathcal{C}_{\epsilon}$ is nonempty for every $\epsilon>0$ if $A$ is chosen large enough and $\alpha, \delta$ are chosen small enough. We check that $\mathcal{C}_{\epsilon}$ is a closed convex set. For $u_{0}, u_{1} \in \mathcal{C}_{\epsilon}$, let $u_{t}$ be the geodesic homotopy of $u_{0}$ and $u_{1}$. Then by the property of NPC space

$$
d\left(u_{t}(p), u_{t}(q)\right) \leq(1-t) d\left(u_{0}(p), u_{0}(q)\right)+t d\left(u_{1}(p), u_{1}(q)\right)
$$

for any $t \in[0,1]$ and $p, q \in \bar{X}$ with $d_{\bar{X}}(p, q) \leq 1$. This shows that the energy is a convex functional in $L_{\rho}^{2}(\bar{X}, Y)$ and that the Hölder estimates are preserved along geodesics in $L_{\rho}^{2}(\bar{X}, Y)$. Thus $\mathcal{C}_{\epsilon}$ is a convex set. The set $\mathcal{C}_{\epsilon}$ is closed since the energy is lower semicontinuous and so is the Hölder estimate since $L^{2}$ convergence is equivalent to uniform convergence in the presence of a uniform modulus of continuity.

Let $p_{0} \in X, Q \in Y$ and $C_{\epsilon}=\left\{u\left(p_{0}\right): u \in \mathcal{C}_{\epsilon}\right\}$. If there exists $R>$ $0, C_{\epsilon} \cap B_{R}(Q) \neq \emptyset$ for all $\epsilon>0$, then we can find a minimizing sequence which is bounded in $L_{\rho}^{2}(\bar{X}, Y)$ and we can find a harmonic equivariant map (see the proof of [KS2] Theorem 2.1.3 to verify that a bounded minimizing sequence yields a minimizing equivariant map). If for any $R>0$, there exists $\epsilon_{0}>0$ so that $C_{\epsilon}$ lies outside of $B_{R}(Q)$ for all $\epsilon<\epsilon_{0}$, then we show that there exists an equivalence class of rays fixed by $\Gamma$. Let $u_{i} \in \mathcal{C}_{2^{-i}}$ and consider the triangle with vertices $Q, u_{i}\left(p_{0}\right)$ and $u_{j}\left(p_{0}\right)$ for $i<j$. Let $u_{t}$ be the geodesic homotopy of $u_{i}$ and $u_{j}$. Then $u_{t} \in \mathcal{C}_{2^{-i}}$ and hence $u_{t}\left(p_{0}\right) \in C_{2^{-i}}$ which implies that $u_{t}\left(p_{0}\right) \cap B_{R}(Q)=\emptyset$, i.e. the geodesic between $u_{i}\left(p_{0}\right)$ and $u_{j}\left(p_{0}\right)$ does not intersect $B_{R}(Q)$. By the $\operatorname{CAT}(\kappa)$ condition, the segments $\overline{Q u_{i}\left(p_{0}\right)}$ and $\overline{Q u_{j}\left(p_{0}\right)}$ are uniformly close on bounded subsets of $X$. Therefore, this sequence of segments is Cauchy in the topology of uniform convergence, and converges to a ray $\sigma$. Then for each $k=1, \ldots, N$

$$
d\left(\rho\left(\gamma_{k}\right) u_{i}\left(p_{0}\right), u_{i}\left(p_{0}\right)\right)=d\left(u_{i}\left(\gamma_{k}\left(p_{0}\right)\right), u_{i}\left(p_{0}\right)\right) \leq C .
$$

independently of $i$ using the uniform Hölder continuity of $u_{i}$. If we let $\delta(p)=$ $\max _{k=1, \ldots, N} d\left(\rho\left(\gamma_{k}\right) p, p\right)$ then this means that $\delta(\cdot)$ is bounded along segment 
$\overline{Q u_{i}\left(p_{0}\right)}$ for each $i$. Therefore, $\delta(\cdot)$ is bounded along $\sigma$, and thus the equivalence class of $\sigma$ is invariant under $\Gamma$.

We also have the following uniqueness theorem:

Theorem 4.6. Let $X, \Gamma, \rho$ and $Y$ as above with $\operatorname{curv}(Y)<\kappa<0$. If $f_{0}, f_{1}$ : $\bar{X} \rightarrow Y$ are $\rho$-equivariant energy minimizing maps, then $f_{0} \equiv f_{1}$ unless $f_{0}(M)$ is contained in a geodesic.

Proof. The uniqueness theorem of harmonic maps when the target has strictly negative curvature is proven in [Me]. It is not too hard to extend the proof there to cover the situation when the target is a admissible Riemannian simplicial complex.

\section{The Korevaar-Schoen compactness theorem.}

In this section, we consider compactness theorems for a family of harmonic maps. The compactness of harmonic maps with a Riemannian domain was obtained by Korevaar and Schoen [KS2]. The main difficulty in allowing the domain $X$ to be a simplicial complex is to control the energy near non-manifold points.

Theorem 5.1. Let $\left(Y_{k}, d_{k}\right)$ be a sequence of NPC spaces, $\rho_{k}: \Gamma \rightarrow \operatorname{Isom}\left(Y_{k}\right)$ a sequence of isometric actions and $f_{k}: \bar{X} \rightarrow\left(Y_{k}, d_{k}\right)$ a sequence of $\rho_{k}$ equivariant harmonic maps so that $E^{f_{k}}=1$. Then (1) there exists a NPC space $\left(Y_{\infty}, d_{\infty}\right)$, an isometric action $\rho_{\infty}: \Gamma \rightarrow \operatorname{Isom}\left(Y_{\infty}\right)$, a nonconstant $\rho_{\infty}$-equivariant map $f_{\infty}: \bar{X} \rightarrow\left(Y_{\infty}, d_{\infty}\right)$ and a subsequence $f_{k^{\prime}}$ so that $f_{k^{\prime}}$ converges locally uniformly to $f_{\infty}$ (see definition 3.3 of [KS2]); in particular, $d_{k^{\prime}}\left(f_{k^{\prime}}(\cdot), f_{k^{\prime}}(\cdot)\right)$ converges uniformly to $d_{\infty}\left(f_{\infty}(\cdot), f_{\infty}(\cdot)\right)$ on any compact subset $K$ of $\bar{X}$ and $f_{k^{\prime}}$ and $f_{\infty}$ satisfies the same modulus of continuity estimates, (2) the energy density functions of $f_{k^{\prime}}$ converges a.e. to that of $f_{\infty}$ and (3) $f_{\infty}$ is harmonic.

Proof. (of (1) and (2)) Since $E^{f_{k}}=1,\left\{f_{k}\right\}$ has a uniform modulus of continuity by Theorem 3.12. Following the proof of [KS2] Proposition 3.7, we can show there exists a subsequence of $\left\{f_{k}\right\}$ (which we will still denote by $f_{k}$ for simplicity) which converges locally uniformly in the pullback sense to a limit $\operatorname{map} f_{\infty}: \bar{X} \rightarrow\left(Y_{\infty}, d_{\infty}\right)$.

We now show that $f_{\infty}$ is a non-constant map. 
Fix $k$ and $v \in V$ and let

$$
E_{k}(r)=\int_{B_{r}(v)}\left|\nabla f_{k}\right|^{2} \text { and } I_{k}(r)=\int_{\partial B_{r}(v)} d_{k}^{2}\left(f_{k}, f_{k}(v)\right) d s
$$

for $0<r \leq 1$. Now fix $r$ and we treat the following two cases:

CASE $1: \frac{r E_{k}(r)}{I_{k}(r)} \geq 1$.

By Corollary 3.5,

$$
\frac{E_{k}(r)}{r^{2}} \leq \frac{E_{k}(1)}{1^{2}}
$$

which implies $E_{k}(r) \leq r^{2} E_{k}(1) \leq r^{2} E^{f_{k}}=r^{2}$.

CASE $2: \frac{r E_{k}(r)}{I_{k}(r)}<1$.

By Theorem $3.12, I_{k}(r) \leq C_{v} r A^{2} r^{2 \alpha}$ where $C_{v} r=\operatorname{vol}\left(\partial B_{r}(v)\right)$. Thus,

$$
E_{k}(r)<\frac{I_{k}(v)}{r} \leq C_{v} A^{2} r^{2 \alpha} \leq C A^{2} r^{2 \alpha}
$$

where $C=\max _{v \in V} C_{v}$.

Thus, CASE 1 and CASE 2 imply that for any $k, v \in V$ and $0<r<1$,

$$
E_{k}(r) \leq r^{2}+C A^{2} r^{2 \alpha}
$$

and for any $k$ and $0<r<1$,

$$
\begin{aligned}
1 & =E^{f_{k}} \\
& =\int_{X-\cup_{v \in V} B_{r}(v)}\left|\nabla f_{k}\right|^{2} d \mu+\int_{\cup_{v \in V} B_{r}(v)}\left|\nabla f_{k}\right|^{2} d \mu \\
& \leq \int_{X-\cup_{v \in V} B_{r}(v)}\left|\nabla f_{k}\right|^{2} d \mu+(\# V)\left(r^{2}+C A^{2} r^{2 \alpha}\right)
\end{aligned}
$$

where $\# V$ is the number of vertices of $X$. Thus,

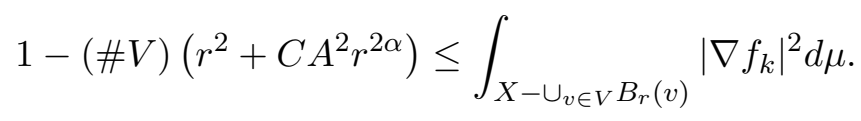

Let $F$ be a face with vertices $v_{1}, v_{2}, v_{3}$ and edges $e_{1}, e_{2}, e_{3}$. Denote the $\epsilon$ neighborhood of $e_{i}$ by $e_{i, \epsilon}$. For $0<\epsilon<r$, let $\Omega$ be smooth domain compactly contained in $F$ so that $F-\Omega \subset \cup_{i=1}^{3} e_{i, \epsilon}$. By [KS2] Theorem 3.11,

$$
\lim _{k \rightarrow \infty} \int_{\Omega}\left|\nabla f_{k}\right|^{2}=\int_{\Omega}\left|\nabla f_{\infty}\right|^{2}
$$


By Theorem 3.11, the Lipschitz constant of $f_{k}$ in $X-\cup_{v \in V} B_{r}(v)$ is uniformly bounded independent of $k$, say by $L$. Therefore,

$$
\begin{aligned}
\limsup _{k \rightarrow \infty} \int_{F-\cup_{i=1}^{3} B_{r}(v)}\left|\nabla f_{k}\right|^{2} & \leq \limsup _{k \rightarrow \infty} \int_{\Omega-\cup_{i=1}^{3} B_{r}(v)}\left|\nabla f_{k}\right|^{2}+3 L \epsilon \\
& \leq \lim _{k \rightarrow \infty} \int_{\Omega}\left|\nabla f_{k}\right|^{2}+3 L \epsilon \\
& =\int_{\Omega}\left|\nabla f_{\infty}\right|^{2}+3 L \epsilon \\
& \leq \int_{F}\left|\nabla f_{\infty}\right|^{2}+3 L \epsilon .
\end{aligned}
$$

Since $\epsilon$ can be chosen arbitrarily small, we see that

$$
\limsup _{k \rightarrow \infty} \int_{X-\cup_{v \in V} B_{r}(v)}\left|\nabla f_{k}\right|^{2} \leq \int_{X}\left|\nabla f_{\infty}\right|^{2} .
$$

Combined with (5.1), we obtain

$$
1-(\# V)\left(r^{2}+C A^{2} r^{2 \alpha}\right) \leq \int_{X}\left|\nabla f_{\infty}\right|^{2} d \mu
$$

Letting $r \rightarrow 0$ and using lower semicontinuity of energy (cf. [KS1] Theorem 1.6.1), we conclude $E^{f_{\infty}}=1$. This shows $f_{\infty}$ is a nonconstant map and finishes the proof of (1). The assertion of (2) follows immediately by the fact that there is no loss of energy and the lower semicontinuity of energy. We defer the proof of (3) to Section 7 .

We also have a compactness theorem of harmonic maps in the case of compact domains.

Theorem 5.2. Fix $B_{1}=D_{1}$ or $B_{1}=X_{1}$ or $B_{1}=X_{1}^{\prime}$ (cf. Section 2.2). Let $B_{\tau}=\left\{z=\left(x_{i}, y_{i}\right) \in B_{1}:|z|=\sqrt{x_{i}^{2}+y_{i}^{2}} \leq \tau\right\}$. Let $\left(Y_{k}, d_{k}\right)$ be a sequence of NPC spaces, $f_{k}: B_{1} \rightarrow\left(Y_{k}, d_{k}\right)$ a sequence of harmonic maps with

$$
I_{k}(1)=\int_{\partial B_{1}} d_{k}^{2}\left(f_{k}(x), f_{k}(0)\right) d s=1
$$

and $E^{f_{k}} \leq C$. Then (1) there exists an NPC space $\left(Y_{\infty}, d_{\infty}\right)$, a non-constant map $f_{\infty}: B_{1} \rightarrow Y_{\infty}$ and a subsequence $f_{k^{\prime}}$ so that $f_{k^{\prime}}$ converges locally uniformly to $f_{\infty}$ (see definition 3.3 of [KS2]), (2) for $0<\tau<1$, the energy density functions of $\left.f_{k^{\prime}}\right|_{B_{\tau}}$ converges a.e. to that of $f_{\infty}$ and (3) for $0<\tau<1$, $f_{\infty}: B_{\tau} \rightarrow Y_{\infty}$ is harmonic. 
Proof. (of (1) and (2)) For the case when $B_{1}=D_{1}$, the statement was proved by [Su]. For any $\tau<1$, the Lipschitz constant of $u_{k}$ in uniformly bounded in $B_{\tau}$ by Theorem 3.11. Therefore there exists a subsequence of $f_{k}$ which converges locally uniformly in the pullback sense in $B_{\tau}$ to a harmonic map by applying the argument of [KS2] Proposition 3.7. By choosing a sequence $\tau_{k} \rightarrow 1$ and by a diagonalization procedure, we can pick a subsequence $f_{k^{\prime}}$ of $f_{k}$ which converges locally uniformly to a map $f_{\infty}$. We now show that $f_{\infty}$ is non-constant. Let

$$
I_{k}(r)=\int_{\partial B_{r}(0)} d_{k}^{2}\left(f_{k}, f_{k}(0)\right) d s
$$

and

$$
E_{k}(r)=\int_{B_{r}(0)}\left|\nabla f_{k}\right|^{2} d \mu
$$

For $\theta \in(0,1)$ and $r_{0} \in(\theta, 1]$, we have

$$
\begin{aligned}
I_{k}\left(r_{0}\right)-I_{k}(\theta)= & \int_{\theta}^{r_{0}} \frac{d}{d \sigma} I_{k}(\sigma) d \sigma \\
= & \int_{\theta}^{r_{0}} \frac{d}{d \sigma} \int_{\partial B_{\sigma}(0)} d^{2}\left(f_{k}, f_{k}(0)\right) d s d \sigma \\
= & \int_{\theta}^{r_{0}} \int_{\partial B_{\sigma}(0)} 2 d\left(f_{k}, f_{k}(0)\right) \frac{d}{d \sigma} d\left(f_{k}, f_{k}(0)\right) d s d \sigma \\
& +\frac{1}{\sigma} \int_{\theta}^{r_{0}} \int_{\partial B_{\sigma}(0)} d^{2}\left(f_{k}, f_{k}(0)\right) d s
\end{aligned}
$$

By the Cauchy-Schwartz inequality and $\frac{d}{d \sigma} d\left(f_{k}, f_{k}(0)\right) \leq\left|\nabla f_{k}\right|^{2}$, we have

$$
I_{k}\left(r_{0}\right)-I_{k}(\theta) \leq \epsilon E_{k}\left(r_{0}\right)+\left(\frac{1}{\epsilon}+\frac{1}{\theta}\right) \int_{\theta}^{r_{0}} I_{k}(r) d r
$$

for $0<\theta<r<1$ and any $\epsilon>0$. By Proposition 3.2,

$$
\frac{r_{0} E_{k}\left(r_{0}\right)}{I_{k}\left(r_{0}\right)} \leq \frac{E_{k}(1)}{I_{k}(1)}=C
$$

and hence

$$
I_{k}\left(r_{0}\right)-I_{k}(\theta) \leq \frac{\epsilon C I_{k}\left(r_{0}\right)}{r_{0}}+\left(\frac{1}{\epsilon}+\frac{1}{\theta}\right) \int_{\theta}^{r_{0}} I_{k}(r) d r .
$$


For any $\theta \in\left[\frac{1}{2}, 1\right)$, pick $r_{0} \in(\theta, 1]$. Then $r_{0} \geq \theta \geq \frac{1}{2}$ and by choosing $\epsilon=\frac{1}{4 C}$,

$$
\begin{aligned}
\frac{1}{2} I_{k}\left(r_{0}\right)-I_{k}(\theta) & \leq\left(4 C+\frac{1}{\theta}\right) \int_{\theta}^{r_{0}} I_{k}(r) d r \\
& \leq(4 C+2) \int_{\theta}^{1} I_{k}(r) d r \\
& \leq(4 C+2)(1-\theta) \sup _{r \in[\theta, \sigma]} I_{k}(r) .
\end{aligned}
$$

Since $r_{0}$ is an arbitrary point in $(\theta, 1]$, we have

$$
\frac{1}{2} \sup _{r \in[\theta, 1]} I_{k}(r)-I_{k}(\theta) \leq(4 C+2)(1-\theta) \sup _{r \in[\theta, \sigma]} I_{k}(r) .
$$

Now choose $\theta$ sufficiently close to 1 so that $(4 C+2)(1-\theta) \leq \frac{1}{6}$. Then

$$
I_{k}(\theta) \geq \frac{1}{3} \sup _{r \in[\theta, 1]} I_{k}(r) \geq \frac{1}{3} I(1)=\frac{1}{3}
$$

By the uniform convergence of $d_{k}\left(f_{k}(\cdot), f_{k}(\cdot)\right)$ to $d_{\infty}\left(f_{\infty}(\cdot), f_{\infty}(\cdot)\right)$, we then have

$$
I_{\infty}(\theta) \geq \frac{1}{3}
$$

and this shows that $f_{\infty}$ is not constant.

Next, we prove the convergence of the energies. We consider the case when 0 is a vertex since the proof is simpler if 0 is not a vertex. For any $0<\tau<1$ and $0<r<\tau, f_{k}$ is uniformly Lipschitz continuous in $B_{\tau}-B_{r}$. Thus, following the proof of Theorem 5.1, we can show that the Sobolev energy and directional energy densities of $f_{k}$ converge to that of $f_{\infty}$ in $B_{\tau}-B_{r}$. Using the energy estimate near a vertex of Theorem 5.1, we see that

$$
\begin{aligned}
E_{\infty}(\tau) & \leq \liminf _{k \rightarrow \infty} E_{k}(\tau) \\
& \leq \liminf _{k \rightarrow \infty} E_{k}(\tau)-E_{k}(r)+\left(r^{2}+C A^{2} r^{2 \alpha}\right) \\
& =E_{\infty}(\tau)-E_{\infty}(r)+\left(r^{2}+C A^{2} r^{2 \alpha}\right)
\end{aligned}
$$

Since $r$ can be chosen arbitrarily small, we see that $E^{f_{k}}(\tau) \rightarrow E^{f_{\infty}}(\tau)$. Since there is no loss of total energy in $B_{1-\epsilon}$, the convergence of the energy density functions follows by the lower semicontinuity of energy. We defer the proof of (3) to Section 7 


\section{Tangent maps.}

The tangent map of $f$ is defined to be a limit of the blow up maps defined below.

Definition 6.1. Suppose $f: X \rightarrow Y$ is a map from a flat admissible locally finite 2-complex $X$ to a $\operatorname{NPC}$ space $(Y, d)$. For $p_{0} \in X$ and $\sigma<i n j\left(p_{0}\right)$, let

$$
\begin{gathered}
E_{p_{0}}^{f}(\sigma)=\int_{B_{\sigma}\left(p_{0}\right)}|\nabla f|^{2} d \mu \\
I_{p_{0}}^{f}(\sigma)=\int_{\partial B_{\sigma}\left(p_{0}\right)} d^{2}(f, f(p)) d s
\end{gathered}
$$

and

$$
\mu_{p_{0}}^{f}(\sigma)=\left(I_{p_{0}}^{f}(\sigma) \sigma^{-1}\right)^{-\frac{1}{2}}
$$

If $p_{0}$ is a face point, let $B_{1}$ be the unit disk $D_{1}$. If $p_{0}$ is an edge point, let $B_{1}$ be an edge piece $X_{1}$ isometric to $B_{\sigma}\left(p_{0}\right)$. If $p_{0}$ is a vertex point, let $B_{1}$ be an vertex piece $X_{1}^{\prime}$ isometric to $B_{\sigma}\left(p_{0}\right)$. Let $d_{\sigma, p_{0}}$ be the distance function on $Y$ defined by $d_{\sigma, p_{0}}(\cdot, \cdot)=\mu_{p_{0}}^{f}(\sigma) d(\cdot, \cdot)$. We define the $\sigma$-blow up map of $f$ at $p_{0}$ as the map

$$
{ }^{\sigma} f: B_{1} \rightarrow\left(Y, d_{\sigma, p_{0}}\right)
$$

defined by

$$
{ }^{\sigma} f(z)=f\left(\frac{z}{\sigma}\right)
$$

where $B_{\sigma}\left(p_{0}\right)$ is identified with $D_{\sigma}, X_{\sigma}$ or $X_{\sigma}^{\prime}$ via $z=z(p)$ defined in Section 2 .

Proposition 6.2. Let $f: X \rightarrow(Y, d)$ be a harmonic map from a flat admissible 2-complex into a NPC space $(Y, d)$. For each $p_{0} \in X$, let $B_{1}=D_{1}$ if $p_{0}$ is a face point, $B_{1}=X_{1}$ if $p_{0}$ is an edge point and $B_{1}=X_{1}^{\prime}$ if $p_{0}$ is a vertex point and let

$$
\alpha=\lim _{\sigma \rightarrow 0} \frac{\sigma E_{p_{0}}^{f}(\sigma)}{I_{p_{0}}^{f}(\sigma)} .
$$

There exists a sequence $\sigma_{i} \rightarrow 0$ so that the $\sigma_{i}$-blow up maps ${ }^{\sigma_{i}} f: B_{1} \rightarrow\left(Y, d_{\sigma, p_{0}}\right)$ of $f$ at $p_{0}$ converge in the sense of Theorem 5.2 to a map $f_{*}: B_{1} \rightarrow\left(Y_{*}, d_{*}\right)$ into a NPC space. The map $f_{*}$ is Lipschitz continuous except possibly at the vertex and is homogeneous of order $\alpha$, i.e.

$$
d_{*}\left(f_{*}(z), f_{*}(0)\right)=|z|^{\alpha} d_{*}\left(f_{*}\left(\frac{z}{|z|}\right), f(0)\right)
$$

for every $z \in B_{1}$. We call $f_{*}$ a tangent map of $f$ at $p_{0}$. 
Proof. We have

$$
\begin{aligned}
I_{0}^{\sigma f}(1) & =\left(\mu_{p_{0}}^{f}(\sigma)\right)^{2} \int_{\partial B_{1}\left(p_{0}\right)} d^{2}(f(\sigma x), f(0)) d s \\
& =\left(\mu_{p_{0}}^{f}(\sigma)\right)^{2} \int_{\partial B_{\sigma}\left(p_{0}\right)} d^{2}(f(x), f(0)) \sigma^{-1} d s=1
\end{aligned}
$$

and, by Proposition 3.2,

$$
\begin{aligned}
E_{0}^{\sigma} f(1) & =\left(\mu_{p_{0}}^{f}(\sigma)\right)^{2} \int_{B_{1}} \sigma^{2}|\nabla f|^{2}(\sigma x) d x \\
& =\left(\mu_{p_{0}}^{f}(\sigma)\right)^{2} \int_{B_{\sigma}}|\nabla f|^{2}(x) d x \\
& =\frac{\sigma E_{0}^{f}(\sigma)}{I_{0}^{f}(\sigma)} \leq \frac{E_{0}^{f}(1)}{I_{0}^{f}(1)} \leq E_{0}
\end{aligned}
$$

for any $\sigma<1$. Therefore, Theorem 5.2 implies that there exists $\sigma_{i} \rightarrow 0$, so that ${ }^{\sigma_{i}} f: B_{1} \rightarrow\left(T, d_{\sigma_{i}}\right)$ converges locally uniformly in the pull back sense to a limit map $f_{*}: B_{1} \rightarrow\left(T_{*}, d_{*}\right)$. The map $f_{*}$ is Lipschitz continuous in a small neighborhood $U$ of a non-vertex point since the Lipschitz constant of ${ }^{\sigma_{i}} f$ in $U$ is independent of $i$.

To see that $f_{*}$ is homogeneous, we will follow the argument of [GS] Lemma 3.2. But we need to work with the fact that we have not yet shown the harmonicity of $f_{*}$. First, observe

$$
\begin{aligned}
\frac{\tau E_{0}^{f_{*}}(\tau)}{I_{0}^{f^{*}}(\tau)} & =\lim _{\sigma_{i} \rightarrow 0} \frac{\tau E_{0}^{\sigma_{i}} f(\tau)}{I_{0}^{\sigma_{i}} f(\tau)} \\
& =\lim _{\sigma_{i} \rightarrow 0} \frac{\tau\left(\mu_{p_{0}}^{f}\left(\sigma_{i}\right)\right)^{-2} E_{p_{0}}^{f}\left(\tau \sigma_{i}\right)}{\left(\mu_{p_{0}}^{f}\left(\sigma_{i}\right)\right)^{-2} \sigma_{i}^{-1} I_{p_{0}}^{f}\left(\tau \sigma_{i}\right)} \\
& =\lim _{\sigma_{i} \rightarrow 0} \frac{\tau \sigma_{i} E_{p_{0}}^{f}\left(\tau \sigma_{i}\right)}{I_{p_{0}}^{f}\left(\tau \sigma_{i}\right)} \\
& =\alpha .
\end{aligned}
$$

Using equality (3.2) with ${ }^{\sigma_{i}} f$ replaced with $f$ and the convergence of the energy density functions, we see that

$$
0=\int_{B_{r}\left(p_{0}\right)} \frac{\partial \zeta}{\partial x} x\left(\frac{\partial f_{*}}{\partial x}\right)^{2}+\frac{\partial \zeta}{\partial y} y\left(\frac{\partial f_{*}}{\partial y}\right)^{2}+2 \frac{\partial \zeta}{\partial x} y \frac{\partial f_{*}}{\partial x} \frac{\partial f_{*}}{\partial y}+2 \frac{\partial \zeta}{\partial y} x \frac{\partial f_{*}}{\partial x} \frac{\partial f_{*}}{\partial y} .
$$


Taking $\zeta$ to be an approximation of the characteristic function of the ball $B_{r}\left(p_{0}\right)$, we get

$$
\left.\frac{d}{d r}\left(E_{0}^{f_{*}}(r)\right)\right)=2 \int_{\partial B_{r}(0)}\left|\frac{\partial f_{*}}{\partial r}\right|^{2} d s .
$$

Additionally, Proposition 3.1 with $f$ replaced by ${ }^{\sigma_{i}} f$ and $\varphi$ a radial function which is equal to 0 outside $B_{r}(0)$ and equal to 1 near 0 implies that

$$
\begin{aligned}
& -\int_{B_{r}(0)} \frac{\partial}{\partial r} d\left(f^{*}, f^{*}(0)\right) \frac{\partial \varphi}{\partial r} d \mu \\
& =\int_{B_{r}(0)} d\left(f^{*}, f^{*}(0)\right) \frac{\partial^{2} \varphi}{\partial r^{2}} d \mu \\
& =\lim _{\sigma \rightarrow 0} \int_{B_{r}(0)} d\left({ }^{\sigma_{i}} f,^{\sigma_{i}} f(0)\right) \frac{\partial^{2} \varphi}{\partial r^{2}} d \mu \\
& =-\lim _{\sigma \rightarrow 0} \int_{B_{r}(0)} \frac{\partial}{\partial r} d\left({ }^{\sigma_{i}} f,^{\sigma_{i}} f(0)\right) \frac{\partial \varphi}{\partial r} d \mu \\
& =-\int_{B_{r}(0)} \nabla d\left({ }^{\sigma_{i}} f,^{\sigma_{i}} f(0)\right) \cdot \nabla \varphi \\
& \geq 2 \int_{B_{r}(0)}\left|\nabla^{\sigma_{i}} f\right|^{2} \varphi d \mu \\
& =2 \int_{B_{r}(0)}\left|\nabla f_{*}\right|^{2} \varphi d \mu .
\end{aligned}
$$

Letting $\varphi$ approximate the characteristic function of $B_{\sigma}(0)$, we obtain

$$
\int_{B_{r}(0)}\left|\nabla f_{*}\right|^{2} d \mu \leq \int_{\partial B_{r}(0)} d\left(f_{*}, f_{*}(0)\right) \frac{\partial}{\partial r} d\left(f_{*}, f_{*}(0)\right) d s .
$$

Thus, following the proof of Proposition 3.2, we conclude that

$$
\begin{aligned}
\frac{d}{d r} \log & \left(\frac{I_{0}^{f_{*}}(r)}{r E_{0}^{f_{*}}(r)}\right) \\
\leq & 2\left(E_{0}^{f_{*}}(r) I_{0}^{f_{*}}(r)\right)^{-1}\left[\left(\int_{\partial B_{r}(0)} d\left(f_{*}, f_{*}(0)\right) \frac{\partial}{\partial r} d\left(f^{*}, f^{*}(0)\right) d s\right)^{2}\right. \\
& \left.-\left(\int_{\partial B_{r}(0)} d^{2}\left(f_{*}, f_{*}(0)\right) d s\right)\left(\int_{\partial B_{r}(0)}\left|\frac{\partial f^{*}}{\partial r}\right|^{2} d s\right)\right] \leq 0
\end{aligned}
$$


Therefore, by (6.1),

$$
\begin{aligned}
& \left(\int_{\partial B_{r}(0)} d\left(f_{*}, f_{*}(0)\right) \frac{\partial}{\partial r} d\left(f^{*}, f^{*}(0)\right) d s\right)^{2} \\
& \quad=\left(\int_{\partial B_{r}(0)} d^{2}\left(f_{*}, f_{*}(0)\right) d s\right)\left(\int_{\partial B_{r}(0)}\left|\frac{\partial f^{*}}{\partial r}\right|^{2} d s\right)
\end{aligned}
$$

Combined with (3.4) and (3.5) with $f$ replaced by $f_{*}$, we have that

$$
\frac{\partial}{\partial r} d\left(f_{*}, f_{*}(0)\right) d s=\left|\frac{\partial f_{*}}{\partial r}\right|
$$

almost everywhere. For a ray $\gamma(r)=r \xi, \xi \in \partial B_{1}(0)$ and $r_{0} \leq r \leq 1$,

$$
\begin{aligned}
\operatorname{length}\left(f_{*}(\gamma)\right) & =\int_{r_{0}}^{1}\left|\frac{\partial f_{*}}{\partial r}\right| d s \\
& =\int_{r_{0}}^{1} \frac{\partial}{\partial r} d\left(f_{*}, f_{*}(0)\right) d s \\
& =d\left(f_{*}(\xi), f_{*}(0)\right)-d\left(f_{*}\left(r_{0} \xi\right), f_{*}(0)\right) \\
& \leq d\left(f_{*}(\xi), f_{*}\left(r_{0} \xi\right)\right),
\end{aligned}
$$

which implies that $f_{*}(\gamma)$ is a geodesic path in $Y_{*}$. Additionally, (6.3) and (3.4) with $f$ replaced by $f_{*}$ implies that there exists a constant $h(r)$ so that

$$
\left|\frac{\partial f_{*}}{\partial r}\right|=h(r) d\left(f_{*}, f_{*}(0)\right)
$$

Combined with the equality in (6.2), we obtain

$$
E_{0}^{f_{*}}(r)=\int_{\partial B_{r}(0)} d\left(f_{*}, f_{*}(0)\right)\left|\frac{\partial f_{*}}{\partial r}\right| d s=h(r) I_{0}^{f_{*}}(r) .
$$

On the other hand, we have that $E_{0}^{f_{*}}(r)=\alpha r^{-1} I_{0}^{f_{*}}(r)$ so we conclude $h(r)=$ $\alpha r^{-1}$. Integration of

$$
\frac{\frac{\partial}{\partial r} d\left(f_{*}, f_{*}(0)\right)}{d\left(f_{*}, f_{*}(0)\right)}=h(r)=\frac{\alpha}{r}
$$

along a ray from $z$ to $\frac{z}{|z|}$ implies the homogeneity.

With the help of the order function and tangent map, we can make the following regularity estimate of a harmonic map $f: X \rightarrow Y$ on a face $F$ of $\tilde{X}$. This gives the explicit dependency of the Lipschitz constant in terms of the distance to a vertex. 
Theorem 6.3. For a vertex $v$ of $F$, let

$$
\alpha=\lim _{r \rightarrow 0} \frac{r E_{v}(r)}{I_{v}(r)}
$$

where $E_{v}(r)=\int_{B_{r}(v)}|\nabla f|^{2} d \mu$ and $I_{v}(r)=\int_{\partial B_{r}(v)} d^{2}(f, f(v)) d \mu$. Then

$$
|\nabla f|^{2}(z) \leq C r^{2 \alpha-2}
$$

for all $z \in B_{\frac{1}{2}}(v)$ where $C$ depends on $E^{f}$ and $r=d_{X}(v, z)$.

Proof. It will be sufficient to estimate the gradient bound in $X_{\frac{1}{2}}^{\prime} \subset X_{1}^{\prime}$ for a harmonic map $f: X_{1}^{\prime} \rightarrow T$ dependent only on $E^{f}$ and the distance to the vertex $v=(0,0)$. For any point $\left(x_{i}, y_{i}\right) \in X_{1}^{\prime}-\{v\}$, let $\alpha\left(x_{i}, y_{i}\right)$ be the order of $f$ at $\left(x_{i}, y_{i}\right)$, i.e.

$$
\alpha\left(x_{i}, y_{i}\right)=\lim _{r \rightarrow 0} \frac{r E_{\left(x_{i}, y_{i}\right)}(r)}{I_{\left(x_{i}, y_{i}\right)}(r)} .
$$

Since $f_{*}$ is a homogeneous map of order $\alpha\left(x_{i}, y_{i}\right)$, the Lipschitz continuity of $f_{*}$ implies that $\alpha\left(x_{i}, y_{i}\right) \geq 1$. In particular, Proposition 3.2 implies that for any point $\left(x_{i}, y_{i}\right) \in X_{1}^{\prime}-\{v\}$,

$$
\begin{aligned}
& \left(\frac{E_{\left(x_{i}, y_{i}\right)}(r)}{r^{2}}\right)^{\prime} \geq 0 \\
& \left(\frac{I_{\left(x_{i}, y_{i}\right)}(r)}{r^{3}}\right)^{\prime} \geq 0
\end{aligned}
$$

and

$$
\frac{I_{\left(x_{i}, y_{i}\right)}(r)}{r} \leq E_{\left(x_{i}, y_{i}\right)}(r)
$$

Let $z_{0}=\left(x_{i}, y_{i}\right) \in X_{\frac{1}{2}}^{\prime}$ be a face point and $r_{0}=\left|z_{0}\right|$. We may assume without the loss of generality that $\tan ^{-1}\left(\frac{y_{i}}{x_{i}}\right) \leq \frac{\pi}{6}$ (otherwise, we can reparametrize $W_{i} \subset X_{1}^{\prime}$ so that the line $\tan ^{-1}\left(\frac{y}{x}\right)=\frac{\pi}{3}$ is the $x$-axis and vice versa). Thus, $y_{i} \leq \frac{x_{i}}{\sqrt{3}}$. Let $\delta=x_{i} \sin \frac{\pi}{3}=\frac{2 x_{i}}{\sqrt{3}}$ and note that $B_{\delta}\left(x_{i}, 0\right) \subset \operatorname{st}\left(x_{i}, y_{i}\right)$ and $2 y_{i} \leq \frac{2 x_{i}}{\sqrt{3}}=\delta$. Let $\sigma<y_{i}$ and $\zeta \in B_{\frac{\sigma}{2}}\left(z_{0}\right)$. By Proposition 3.8,

$$
d^{2}\left(f(\zeta), f\left(x_{i}, y_{i}\right)\right) \leq \frac{c}{\sigma} \int_{\partial B_{\sigma}\left(z_{i}, y_{i}\right)} d^{2}\left(f, f\left(x_{i}, y_{i}\right)\right) d s=c \frac{I_{\left(x_{i}, y_{i}\right)}(\sigma)}{\sigma}
$$


Furthermore,

$$
\begin{aligned}
\frac{I_{\left(x_{i}, y_{i}\right)}(\sigma)}{\sigma} & \leq \frac{I_{\left(x_{i}, y_{i}\right)}\left(y_{i}\right)}{y_{i}^{3}} \sigma^{2}(\text { by inequality }(6.6)) \\
& \leq \frac{E_{\left(x_{i}, y_{i}\right)}\left(y_{i}\right)}{y_{i}^{2}} \sigma^{2}(\text { by inequality }(6.7)) \\
& \leq 4 \frac{E_{\left(x_{i}, 0\right)}\left(2 y_{i}\right)}{\left(2 y_{i}\right)^{2}} \sigma^{2}\left(\text { since } B_{y_{i}}\left(x_{i}, y_{i}\right) \subset B_{2 y_{i}}\left(x_{i}, 0\right)\right) \\
& \leq 4 \frac{E_{\left(x_{i}, 0\right)}(\delta)}{\delta^{2}} \sigma^{2}(\text { by inequality }(6.5)) \\
& \leq 12 \frac{E_{(0,0)}\left(2 x_{i}\right)}{\left(2 x_{i}\right)^{2}} \sigma^{2}\left(\text { since } B_{\delta}\left(x_{i}, 0\right) \subset B_{2 x_{i}}(0,0)\right)
\end{aligned}
$$

By Proposition 3.2 and the assumption that $\alpha(0,0) \geq \alpha$, we have

$$
\frac{E_{(0,0)}\left(2 x_{i}\right)}{\left(2 x_{i}\right)^{2 \alpha}} \leq \frac{E_{(0,0)}(1)}{1^{2 \alpha}}=E^{f}
$$

which implies

$$
\frac{d^{2}\left(f(\zeta), f\left(x_{i}, y_{i}\right)\right)}{\sigma^{2}} \leq 12 c E^{f}\left(2 x_{i}\right)^{2 \alpha-2}
$$

for all $\zeta \in B_{\frac{\sigma}{2}}\left(x_{i}, y_{i}\right)$. Since $\left(2 x_{i}\right)^{2}=4 x_{i}^{2} \geq 3 x_{i}^{2}+3 y_{i}^{2}=3 r_{0}^{2}$,

$$
|\nabla f|^{2}\left(z_{0}\right) \leq C r_{0}^{2 \alpha-2}
$$

where $C$ depends on $E^{f}$.

\section{Completion of the proof of Theorem 5.1 and 5.2.}

In this section, we will prove assertion (3) of Theorem 5.1 and Theorem 5.2; i.e. we show that the limit map $f_{\infty}$ is harmonic in each of the compactness theorems. In order to do this, we essentially repeat the arguments of Theorem 3.9 and Theorem 3.11 resp. of [KS2]. But because we consider a singular domain $\bar{X}$, we must be careful with the mollification estimates used in their proof.

Proof. (of Theorem 5.1 (3)) Let $\eta$ be a smooth, non-increasing function defined on the interval $[0,1]$ which is equal to 0 in the neighborhood of 1 and so that

$$
\int_{\mathbf{R}^{2}} \eta(|x|)=1
$$


Let $\eta_{\epsilon}(t)=\frac{1}{\epsilon^{2}} \eta\left(\frac{t}{\epsilon}\right)$. For a fixed $\epsilon>0$ and $z \in \bar{X}$, define

$$
\eta_{z}: \bar{X} \rightarrow \mathbf{R}^{+} \cap\{0\}
$$

by setting

$$
\eta_{z}(x)=\eta_{\epsilon}(|x-z|) .
$$

Note that for $z \in \bar{X}$, we can only guarantee that the total integral of $\eta_{z}$ is equal to 1 if $z$ is a interior point of a some face $F$ and the distance from $z$ to $\partial F$ is at least $\epsilon$. For $z, w \in \bar{X}$,

$$
\begin{aligned}
\left|\eta_{z}(x)-\eta_{w}(x)\right| & =\left|\eta_{\epsilon}(|x-z|)-\eta_{\epsilon}(|x-w|)\right| \\
& =\left|\int_{|x-z|}^{|x-w|} \eta_{\epsilon}^{\prime}(t) d t\right| \\
& \leq \frac{M}{\epsilon^{3}}|| x-w|-| x-z|| \\
& \leq \frac{M}{\epsilon^{3}}|z-w|
\end{aligned}
$$

where $M=\sup _{t \in[0,1]} \eta^{\prime}(t)$. Additionally, there exists constant $M^{\prime}$ so that

$$
\begin{aligned}
\left|\int_{X} \eta_{z} d \mu-\int_{X} \eta_{w} d \mu\right| & \leq \frac{M}{\epsilon^{3}}|z-w| \cdot \mu\left(\operatorname{spt}\left(\eta_{z}-\eta_{w}\right)\right) \\
& \leq \frac{M^{\prime}}{\epsilon^{3}}|z-w| \cdot(\epsilon+|z-w|)^{2} \\
& \leq \frac{M^{\prime}}{\epsilon}|z-w|
\end{aligned}
$$

for $|z-w|<\epsilon$. Thus, for $|z-w|<\epsilon$,

$$
\begin{aligned}
& \left|\left(\frac{\eta_{z}}{\int_{X} \eta_{z} d \mu}\right)-\left(\frac{\eta_{w}}{\int_{X} \eta_{w} d \mu}\right)\right| \\
& =\frac{1}{\left(\int_{X} \eta_{z} d \mu\right)\left(\int_{X} \eta_{w} d \mu\right)} \\
& \quad \times\left(\left|\eta_{z}-\eta_{w}\right|\left(\int_{X} \eta_{w} d \mu\right)+\eta_{w}\left|\int_{X} \eta_{w} d \mu-\int_{X} \eta_{z} d \mu\right|\right) \\
& \leq \frac{1}{\left(\int_{X} \eta_{z} d \mu\right)\left(\int_{X} \eta_{w} d \mu\right)} \times \frac{M \int_{X} \eta_{w} d \mu+\eta(0) M^{\prime}}{\epsilon^{3}}|z-w| \\
& \leq \frac{M^{\prime \prime}}{\epsilon^{3}}|z-w|
\end{aligned}
$$

where $M^{\prime \prime}$ only depends on $M$ and $M^{\prime}$ and the number of faces of $X$. 
With $(Y, d)$ an NPC space, let $h: X \rightarrow Y$ satisfy

$$
d\left(h\left(x_{1}\right), h\left(x_{2}\right)\right) \leq C t^{\alpha_{0}-1} \epsilon
$$

for $\left|x_{1}-x_{2}\right|<\epsilon<\frac{t}{3}$ with $t$ the distance from $x_{1}$ to $V$ and

$$
d\left(h\left(x_{1}\right), h\left(x_{2}\right)\right) \leq C \epsilon^{2 \alpha_{0}}
$$

for $\left|x_{1}-x_{2}\right|<\epsilon$. Let $\bar{h}(z)$ be the center of mass of the map $h$ with respect to the measure

$$
d \nu_{z}=\left(\frac{\eta_{z}}{\int_{X} \eta_{z} d \mu}\right) d \mu
$$

The existence and uniqueness of $\bar{h}(z)$ follows, for example, from Lemma 2.5.1 of $[\mathrm{KS} 1]$. Set $v_{t}=(1-t) \bar{h}(w)+t \bar{h}(z)$ where we use the notation $(1-t) P+t Q$ to denote the unique point in $X$ which is distance $t d(P, Q)$ away from $P$ and $(1-t) d(P, Q)$ away from $Q$. Then for any $x \in X$,

$$
\begin{aligned}
& d(h(x), \bar{h}(w))+d\left(h(x), v_{t}\right) \\
& \quad \leq d(h(x), \bar{h}(w))+\sqrt{(1-t) d^{2}(h(x), \bar{h}(z))+t d^{2}(h(x), \bar{h}(w))} \\
& \quad \leq 2 d(h(x), \bar{h}(w))+d(h(x), \bar{h}(z)) \\
& \quad \leq 3 d(h(x), \bar{h}(z))+d(\bar{h}(z), \bar{h}(w)) .
\end{aligned}
$$

By Proposition 1.5.1 of [KS2], we have

$$
\begin{aligned}
& d^{2}(\bar{h}(z), \bar{h}(w)) \\
& \quad \leq \frac{1}{2} \limsup _{t \rightarrow 0} \int_{B_{1}(z)} \frac{d^{2}(h(x), \bar{h}(w))-d^{2}\left(h(x), v_{t}\right)}{t}\left|d \nu_{z}-d \nu_{w}\right| \\
& \quad \leq \frac{1}{2} \limsup _{t \rightarrow 0} \int_{B_{1}(z)} \frac{d(h(x), \bar{h}(w))+d\left(h(x), v_{t}\right) t d(\bar{h}(z), \bar{h}(w))}{t}\left|d \nu_{z}-d \nu_{w}\right|,
\end{aligned}
$$

where the triangle inequality was used to derive the second inequality. Dividing both sides by $d(\bar{h}(z), \bar{h}(w))$ and applying (7.4) for the first inequality below and (7.1) for the second, we obtain

$$
\begin{aligned}
& d(\bar{h}(z), \bar{h}(w)) \\
& \quad \leq \frac{1}{2} \int_{B_{1}(z)}(3 d(h(x), \bar{h}(z))+d(\bar{h}(z), \bar{h}(w)))\left|d \nu_{z}-d \nu_{w}\right| \\
& \quad \leq \frac{1}{2} \int_{B_{2 \epsilon}(z)}(3 d(h(x), \bar{h}(z))+d(\bar{h}(z), \bar{h}(w))) \frac{M^{\prime \prime}}{\epsilon^{3}}|z-w| d \mu
\end{aligned}
$$


for $|z-w|<\epsilon$. Here, note that the domain of integration $B_{2 \epsilon}(z)$ in the second integral above comes from the fact that $\operatorname{spt}\left(\nu_{z}-\nu_{w}\right) \subset B_{2 \epsilon}(z)$. By (7.2), if $z$ is a point at a distance at least $3 \epsilon$ from $V$, then for $x \in B_{2 \epsilon}(z)$, there exists a constant $\hat{C}$ so that

$$
d(h(x), \bar{h}(z)) \leq \sup _{y \in B_{\epsilon}(z)} d(h(x), h(y)) \leq \hat{C} t^{\alpha_{0}-1} \cdot 2 \epsilon,
$$

where $t$ is the distance of $z$ from $V$. Hence, by (7.1) and (7.5),

$$
d(\bar{h}(z), \bar{h}(w)) \leq \frac{1}{2} \int_{B_{2 \epsilon}(z)}\left(6 \hat{C} t^{\alpha_{0}-1} \epsilon+d(\bar{h}(z), \bar{h}(w))\right) \frac{M^{\prime \prime}}{\epsilon^{3}}|z-w| d \mu
$$

for $z \in X-\cup_{v \in V} B_{3 \epsilon}(v)$ and $w \in B_{\epsilon}(z)$, which implies

$$
\frac{d(\bar{h}(z), \bar{h}(w))}{|z-w|} \leq \hat{M}\left(6 \hat{C} t^{\alpha_{0}-1}+\frac{d(\bar{h}(z), \bar{h}(w))}{\epsilon}\right)
$$

where $\hat{M}$ is dependent on $M^{\prime \prime}$ and the number of faces of $X$. Furthermore, by (7.3),

$$
d(h(x), \bar{h}(z)) \leq \sup _{y \in B_{\epsilon}(z)} d(h(x), h(y)) \leq C(2 \epsilon)^{\alpha_{0}}
$$

for any $z \in X$ and $x \in B_{2 \epsilon}(z)$. Hence, by (7.5),

$$
d(\bar{h}(z), \bar{h}(w)) \leq \frac{1}{2} \int_{B_{2 \epsilon}(z)}\left(3 C(2 \epsilon)^{\alpha_{0}}+d(\bar{h}(z), \bar{h}(w))\right) \frac{M^{\prime \prime}}{\epsilon^{3}}|z-w| d \mu
$$

for any $z \in X$ and $w \in B_{\epsilon}(z)$. Thus,

$$
\begin{aligned}
\frac{d(\bar{h}(z), \bar{h}(w))}{|z-w|} & \leq \frac{1}{2} \int_{B_{2 \epsilon}(z)} \frac{M^{\prime \prime}}{\epsilon^{3}}\left(3 C(2 \epsilon)^{\alpha_{0}}+d(\bar{h}(z), \bar{h}(w))\right) d \mu \\
& \leq \tilde{M}\left(3 C(2 \epsilon)^{\alpha_{0}-1}+\frac{d(\bar{h}(z), \bar{h}(w))}{\epsilon}\right)
\end{aligned}
$$

where $\tilde{M}$ is dependent on $M^{\prime \prime}$ and the number of faces of $X$. Therefore, denoting $\bar{h}(z)$ by $h * \eta_{\epsilon}(z)$, we obtain

$$
\left|\nabla\left(h * \eta_{\epsilon}\right)\right|^{2}(z) \leq\left\{\begin{array}{l}
C^{\prime} t^{2 \alpha_{0}-2}, \quad \forall z \in X-\cup_{v \in V} B_{3 \epsilon}(v) \\
C^{\prime} \epsilon^{2 \alpha_{0}-2}, \quad \forall z \in \cup_{v \in V} B_{3 \epsilon}(v)
\end{array}\right.
$$

where $t$ is the distance of $z$ to the nearest vertex and the constant $C^{\prime}$ is independent of $\epsilon$. Now consider a $\epsilon$-neighborhood $e_{\epsilon}$ of an edge $e$ inside $B_{3 / 4}\left(v_{0}\right)-$ 
$B_{3 \epsilon}\left(v_{0}\right)$ for some vertex $v_{0}$. We can cover this set by a finite number of strips $\left[2 \epsilon, \frac{3}{4}\right] \times[0, \epsilon]$. Thus, the energy of $h * \eta_{\epsilon}$ in $e_{\epsilon} \cap\left(B_{3 / 4}\left(v_{0}\right)-B_{3 \epsilon}\left(v_{0}\right)\right)$ is bounded by

$$
C^{\prime \prime} \int_{0}^{\epsilon} \int_{2 \epsilon}^{3 / 4} t^{2 \alpha_{0}-2} d t d \tau=C^{\prime \prime} \frac{1}{2 \alpha_{0}-1}\left((3 / 4)^{2 \alpha_{0}-1}-(2 \epsilon)^{2 \alpha_{0}-1}\right) \epsilon
$$

where $C^{\prime \prime}$ is a constant dependent $C^{\prime}$ and on the number of faces incident to $e$. Additionally, the energy in $B_{v_{0}}(3 \epsilon)$ for $v_{0} \in V$ is bounded by

$$
C^{\prime \prime \prime} \epsilon^{2 \alpha_{0}-2} \cdot \epsilon^{2}=C^{\prime \prime \prime} \epsilon^{2 \alpha_{0}}
$$

where $C^{\prime \prime \prime}$ is a constant dependent on $C^{\prime}$ and on the number of faces incident to $v_{0}$. This implies that, we can bound the energy of $h * \eta_{\epsilon}$ in the $\epsilon$-neighborhood $X_{\epsilon}^{(1)}$ of the 1-skeleton of $X$ by $\bar{C}\left(\epsilon+\epsilon^{2 \alpha_{0}}\right)$ for some constant $\bar{C}$ independent of $\epsilon$. By Theorem 1.5.2 of [KS2], the energy of $h * \eta_{\epsilon}$ in $X-X_{\epsilon}^{(1)}$ is bounded by

$$
(1+\tilde{C} \epsilon){ }_{\nu} E_{\epsilon}^{h}
$$

for some constant $\tilde{C}$. Here, ${ }_{\nu} E_{\epsilon}^{h}$ is the $\epsilon$-approximate energy of $h$ as defined in [KS1], [KS2]. In summary, the energy of $h * \eta_{\epsilon}$ has a bound of

$$
E^{h * \eta_{\epsilon}} \leq(1+\tilde{C} \epsilon){ }_{\nu} E_{\epsilon}^{h}+\bar{C}\left(\epsilon+\epsilon^{2 \alpha_{0}}\right) .
$$

Let $h_{\infty}: \bar{X} \rightarrow\left(Y_{\infty}, d_{\infty}\right)$ be a $\rho_{\infty}$-equivariant harmonic map. Then $h_{\infty}$ is globally Hölder continuous and the Lipschitz constant of $h_{\infty}$ in a neighborhood at a distance $t$ away from $V$ is $L t^{\alpha_{0}-1}$ for some constant $L$ and $\alpha_{0}$ by Theorem 3.12 and Theorem 6.3. Thus, for any $\delta>0$ and $k$ sufficiently large, we can construct a piecewise constant map $h_{k}: X \rightarrow Y_{k}$ so that

$$
{ }_{\nu} E_{\epsilon}^{h_{k}}<E^{h_{\infty}}+\delta
$$

and so that inequalities (7.2) and (7.3) are true with $h$ replaced by $h_{k}$ by following the proof of Theorem 3.9 [KS2]. By (7.7) and (7.8) and using the fact that $h_{k} * \eta_{\epsilon}$ is a competitor of $f_{k}$, we get

$$
E^{f_{k}} \leq E^{h_{k} * \eta_{\epsilon}} \leq(1+\tilde{C} \epsilon)\left(E^{h_{\infty}}+\delta\right)+\bar{C}\left(\epsilon+\epsilon^{2 \alpha_{0}}\right) .
$$

Combining this with the lower semicontinuity of energy and noting that $\epsilon$ and $\delta$ can be made arbitrarily small, we obtain

$$
E^{f_{\infty}} \leq \liminf _{k \rightarrow \infty} E^{f_{k}} \leq \limsup _{k \rightarrow \infty} E^{f_{k}} \leq E^{h_{\infty}}
$$


which implies that $f_{\infty}$ is minimizing. Taking $h_{\infty}=f_{\infty}$ above, we conclude that $E^{f_{k}} \rightarrow E^{f_{\infty}}$. The convergence of the energy density measures follows from the lower semicontinuity of energy. This completes the proof of Theorem 5.1.

Proof. (of Theorem $5.2(3)$ ) Let $w_{t}$ be the Dirichlet solution in $B_{t}, t \leq \tau$, with boundary value equal to that of $f_{\infty} \mid B_{t}$. By following the argument of [KS2] Theorem 3.11,

$$
\lim _{t \rightarrow \tau} E^{w_{t}}=E^{w_{\tau}} \leq E^{f_{\infty}}
$$

Let

$$
v_{t}(z)=\left\{\begin{array}{l}
w_{t}(z), \quad z \in B_{t} \\
f_{\infty}(z), \quad z \in B_{\tau}-B_{t} .
\end{array}\right.
$$

By the Lipschitz continuity of $f_{\infty}$ in $B_{\tau}-B_{t}$ and Lemma 4.2, $v_{t}$ is uniformly continuous in $B_{\tau}$. Furthermore, (7.10) implies that

$$
\lim _{t \rightarrow \tau} E^{v_{t}} \leq E^{f_{\infty}}
$$

Fix $\delta>0$ and let $t$ sufficiently close to $\tau$ so that

$$
E^{v}=E^{v_{t}}<E^{f_{\infty}}+\delta
$$

where $v=v_{t}$. For $0<\epsilon<(\tau-t) / 2$ sufficiently small,

$$
{ }_{\nu} E_{\epsilon}^{v}<E^{f_{\infty}}+\delta
$$

where ${ }_{\nu} E_{\epsilon}^{v}$ is the $\epsilon$-approximate energy in $B_{\frac{t+\tau}{2}}$ and $\nu$ as in the proof of Theorem 5.1 (3). Since $v$ is uniformly continuous, we can approximate it closely enough in the $C^{\infty}$ norm to obtain a map $\tilde{v}: B_{\tau} \rightarrow Y_{\infty}$ which is piecewise constant in $B_{t}$, is equal to $f_{\infty}$ in $B_{\tau}-B_{t}$ and

$$
{ }_{\nu} E_{\epsilon}^{\tilde{v}}<E^{f_{\infty}}+\delta
$$

For $k$ sufficiently large, we follow [KS2] to construct a map $\tilde{v}_{k}: B_{\tau} \rightarrow Y_{k}$ so that $\tilde{v}_{k}$ is piecewise constant in $B_{t}$, equal to $f_{k}$ in $B_{\tau}-B_{t}$ and

$$
{ }_{\nu} E_{\epsilon}^{\tilde{v}_{k}}<E^{f_{k}}+\delta
$$

The mollification estimate in the proof of Theorem 5.1 (3) implies

$$
E^{\tilde{v}_{k}} * \eta_{\epsilon} \leq(1+\tilde{C} \epsilon)\left(E^{\infty}+\delta\right)+\bar{C}\left(\epsilon+\epsilon^{2 \alpha_{0}}\right)
$$


where the mollified map is defined in $B_{\tau-\epsilon}$. We apply [KS2] Lemma 3.12 (which can be readily modified to our setting) and follow the rest of the proof of [KS2] Theorem 3.11 to bridge the gap between $f_{k}$ and $\tilde{v}_{k} * \eta_{\epsilon}$ somewhere in the strip $B_{\tau}-B_{\frac{\tau+t}{2}}$. The resulting map $v_{k}$ is a competitor for the $f_{k}$ Dirichlet problem and thus

$$
E^{f_{k}} \leq E^{v_{k}} \leq(1+\tilde{C} \epsilon)\left(E^{\infty}+\delta\right)+\bar{C}\left(\epsilon+\epsilon^{2 \alpha_{0}}\right)+2 \delta+\int_{B_{\tau}-B_{t}}\left|\nabla f_{k}\right|^{2} .
$$

This inequality, which is analogous to (7.9), shows

$$
\limsup _{k \rightarrow \infty} E^{f_{k}} \leq E^{\infty}
$$

which combined with the lower semicontinuity of energy implies that $f_{\infty}$ is harmonic. This completes the proof of Theorem 5.2.

\section{The convergence of equivariant harmonic maps.}

Let $\Gamma$ be a finitely generated group, $X$ a flat admissible finite 2 -complex with normal cover $p: \bar{X} \rightarrow X$ and $\Gamma$ as its transformation group. A homomorphism $\rho: \Gamma \rightarrow S L(2, \mathbf{C})$ is called a $S L(2, \mathbf{C})$-representation of $\Gamma$. Let $\mathcal{R}(\Gamma)$ be the set of $S L(2, \mathbf{C})$-representations of $\Gamma$. An element $\rho \in \mathcal{R}(\Gamma)$ defines a character $\chi_{\rho}: \Gamma \rightarrow \mathbf{C}$ by $\chi_{\rho}(g)=\operatorname{Trace}(\rho(g))$. The character variety of $\Gamma$ is the set of equivalence classes of representations in $\mathcal{R}(\Gamma)$ where $\rho_{1}, \rho_{2} \in \mathcal{R}(\Gamma)$ are equivalent if $\chi_{\rho_{1}}=\chi_{\rho_{2}}$. We note that two irreducible representations are equivalent if and only if they are conjugate (cf. [CuSh]). A sequence $\left\{\rho_{k}\right\}$ is said to converge to infinity in the character variety if $\chi_{\rho_{k}}(g)$ converges to infinity for some $g \in \Gamma$. If we interpret $S L(2, \mathbf{C})$ as the isometry group of $\mathbf{H}^{3}$, then Theorem 4.5 assures the existence of a $\rho$-equivariant harmonic map $u_{\rho}$ from $\bar{X}$ to $\mathbf{H}^{3}$ for each $\rho \in \mathcal{R}(\Gamma)$.

For a map $u: \bar{X} \rightarrow \mathbf{H}^{3}$, let $f: \bar{X} \rightarrow\left(\mathbf{H}^{3}, \frac{1}{\sqrt{E^{u_{k}}}} d_{\mathbf{H}^{3}}\right)$ be the map that is pointwise equal to $u$ but with the distance function on the target space $\mathbf{H}^{3}$ rescaled by a factor of $\frac{1}{\sqrt{E^{u_{k}}}}$. We will refer to $f$ as the rescaled map of $u$.

Lemma 8.1. Suppose that $\left\{\rho_{k}\right\}$ is a sequence of irreducible representations converging to infinity in the character variety of $\Gamma$. If $u_{k}=u_{\rho_{k}}: \bar{X} \rightarrow \mathbf{H}^{3}$ is the associated $\rho_{k}$-equivariant harmonic map, then $E^{u_{k}} \rightarrow \infty$.

Proof. Choose a compact set $K \subset \bar{X}$ containing $x$ and $\gamma x$ for some $x \in \bar{X}$. Suppose $E^{u_{k}} \leq C$ for all $k$ and let $f_{k}$ be the rescaled map of $u_{k}$. Then, by 
Theorem 5.1, there exists a subsequence $f_{k^{\prime}}$ and a harmonic map $f_{\infty}: \bar{X} \rightarrow$ $\left(Y_{\infty}, d_{\infty}\right)$ so that $f_{k^{\prime}}$ converges locally uniformly in the pull back sense to a limit map $f_{\infty}$. In particular, for any $x \in \bar{X}$ and any $\gamma \in \Gamma$,

$$
\begin{aligned}
\inf _{z \in \mathbf{H}^{3}} d_{\mathbf{H}^{3}}\left(\rho_{k}(\gamma) z, z\right) & \leq d_{\mathbf{H}^{3}}\left(\rho_{k}(\gamma) u_{k}(x), u(x)\right) \\
& =d_{\mathbf{H}^{3}}\left(u_{k}(\gamma x), u_{k}(x)\right) \\
& \leq \sqrt{C} \cdot \frac{1}{\sqrt{E^{u_{k}}}} d_{\mathbf{H}^{3}}\left(f_{k}(\gamma x), f_{k}(x)\right) \\
& \rightarrow \sqrt{C} d_{\infty}\left(f_{\infty}(\gamma x), f_{\infty}(x)\right)
\end{aligned}
$$

On the other hand, if $\rho_{k}$ converges to infinity, then

$$
\inf _{z \in \mathbf{H}^{3}} d\left(\rho_{k}(\gamma) z, z\right) \rightarrow \infty
$$

for some $\gamma \in \Gamma$ (cf. [Ka] 10.2). This shows $E^{u_{k}} \rightarrow \infty$.

Theorem 8.2. Let $\left\{\rho_{k}\right\}_{k=1,2, \ldots}$ be a sequence of unbounded irreducible $S L(2, \mathbf{C})$ representations of a finitely generated group $\Gamma$ and $u_{k}: \bar{X} \rightarrow \mathbf{H}^{3}$, $k=1,2, \ldots$, be $\rho_{k}$-equivariant harmonic maps. Let $f_{k}: \tilde{X} \rightarrow Y_{k}$ be the rescaled maps where $Y_{k}=\left(\mathbf{H}^{3}, \frac{1}{\sqrt{E^{u_{k}}}} d_{\mathbf{H}^{3}}\right)$. Then there is a subsequence of $f_{k}$ which converges locally uniformly in the pullback sense to a equivariant harmonic map $f_{\infty}: \tilde{X} \rightarrow(T, d)$ into a $\mathbf{R}$-tree. The tree $T$ is minimal, i.e. it does not contain any proper subtree invariant under the action of $\Gamma$, and the length function of the action of $\Gamma$ on $T$ is in the projective class of the Morgan-Shalen limit of the sequence $\left\{\rho_{k}\right\}$.

Proof. We apply Theorem 5.1 to show the convergence of the subsequence of $f_{k}$ to $f_{\infty}$. That fact that the limit space is a tree $(T, d)$, that $T$ is minimal, and the length function is in the projective class of the Morgan-Shalen limit follows readily from $[\mathrm{DDW} 1]$.

\section{References.}

[BesF] M. Bestvina and M. Feighn Stable actions of groups on real trees. Invent. Math. 121 (1995), 287-321.

[Ch] J. Chen. On energy minimizing mappings between and into singular spaces. Duke Math. J. 79 (1995), 77-99. 
[CuSh] M. Culler and P. Shalen. Varieties of group representations and splittings of 3-manifolds. Ann. of Math. Soc. 350 (1998) 809-849.

[DDW1] G. Daskalopoulos, S. Dostoglou and R. Wentworth. Character varieties and harmonic maps to $\mathbf{R}$-trees. Math. Research Letters 5 (1998), 523-533.

[DDW2] G. Daskalopoulos, S. Dostoglou and R. Wentworth. On the MorganShalen Compactification of the $S L(2, \mathbf{C})$ character varieties of surface groups.

[EF] J. Eells and B. Fuglede. Harmonic maps between Riemannian polyhedra. Cambridge Tracts in Mathematics 142, Cambridge University Press, Cambridge 2001.

[F] B. Fuglede. Hölder continuity of harmonic maps from Riemannian polyhedra to spaces of upper bounded curvature. preprint.

[GS] M. Gromov and R. Schoen. Harmonic maps into singular spaces and p-adic superrigidity for lattices in groups of rank one. IHES Publ. Math. 76 (1992) 165-246.

[Jo] J. Jost. Nonpositive curvature: geometric and anlaytic aspects. ETC Lecture Notes, Birkhaäuser, Berlin 1997.

[Ka] M. Kapovich. Hyperbolic Manifolds and Discrete Groups. Birkhäuser. Boston, 2001.

[KS1] N. Korevaar and R. Schoen. Sobolev spaces and harmonic maps for metric space targets. Communications in Analysis and Geometry 1 (1993), 561-659.

[KS2] N. Korevaar and R. Schoen. Global existence theorem for harmonic maps to non-locally compact spaces. Communications in Analysis and Geometry 5 (1997), 333-387.

[KS3] N. Korevaar and R. Schoen. Global existence theorems for harmonic maps: finite rank spaces and an approach to rigidity for smooth actions. Preprint.

[LePa] G. Levitt and F. Paulin. Geometric Group Actions on Trees. Amer. J. of Math. 119 (1997) 83-102.

[Me] C. Mese. Uniqueness theorems for harmonic maps into metric spaces Comm. in Comtemp. Math. 4 (2002) 725-750. 
[Mo] J. Morgan. Group actions on trees and the compactification of the space of classes of $S O(n, 1)$ representations. Topology 25 (1986) 133.

[MoSh1] J. Morgan and P.B. Shalen. Degenerations of hyperbolic structures, I: valuations, trees and surfaces. Ann. of Math. 120 (1984) 401-476,

[MoSh2] J. Morgan and P.B. Shalen. Degenerations of hyperbolic structures, II: measured laminations in 3-manifolds. Ann. of Math. 127 (1988) 403-456.

[MoSh3] J. Morgan and P.B. Shalen. Degenerations of hyperbolic structures, III: actions of 3-manifold groups on trees and Thurston's compactness theorem. Ann. of Math. 127 (1988) 457-519.

[Mo] C.B. Morrey. Multiple integrals in the calculus of variations, Springer-Verlag NY, 1966.

[Ot] J.P. Otal. Le theorem d'hyperbolisation pour le variétés fibrées de dimension 3. Astérisque 235, Société Mathématique de France, Paris, 1996.

[Se] T. Serbinowski. Boundary regularity of harmonic maps to nonpositively curved metric spaces. Comm. Anal. Geom. 2 (1994) 139-154.

[Su] X. Sun. Regularity of harmonic maps to trees. Amer. J. Math. 125 (2003) 737-771. 